\title{
Habitability of planets on eccentric orbits: Limits of the mean flux approximation
}

\author{
Emeline Bolmont ${ }^{1}$, Anne-Sophie Libert ${ }^{1}$, Jeremy Leconte ${ }^{2,3,4}$, and Franck Selsis ${ }^{5,6}$ \\ ${ }^{1}$ NaXys, Department of Mathematics, University of Namur, 8 Rempart de la Vierge, 5000 Namur, Belgium \\ e-mail: emeline.bolmont@unamur.be \\ 2 Canadian Institute for Theoretical Astrophysics, 60st St George Street, University of Toronto, Toronto, ON, M5S3H8, Canada \\ 3 Banting Fellow \\ ${ }^{4}$ Center for Planetary Sciences, Department of Physical \& Environmental Sciences, University of Toronto Scarborough, Toronto, \\ ON, M1C 1A4, Canada \\ 5 Univ. Bordeaux, LAB, UMR 5804, 33270 Floirac, France \\ ${ }^{6}$ CNRS, LAB, UMR 5804, 33270 Floirac, France
}

Received 4 January 2016 / Accepted 28 April 2016

\begin{abstract}
Unlike the Earth, which has a small orbital eccentricity, some exoplanets discovered in the insolation habitable zone (HZ) have high orbital eccentricities (e.g., up to an eccentricity of $\sim 0.97$ for HD 20782 b). This raises the question of whether these planets have surface conditions favorable to liquid water. In order to assess the habitability of an eccentric planet, the mean flux approximation is often used. It states that a planet on an eccentric orbit is called habitable if it receives on average a flux compatible with the presence of surface liquid water. However, because the planets experience important insolation variations over one orbit and even spend some time outside the $\mathrm{HZ}$ for high eccentricities, the question of their habitability might not be as straightforward. We performed a set of simulations using the global climate model LMDZ to explore the limits of the mean flux approximation when varying the luminosity of the host star and the eccentricity of the planet. We computed the climate of tidally locked ocean covered planets with orbital eccentricity from 0 to 0.9 receiving a mean flux equal to Earth's. These planets are found around stars of luminosity ranging from $1 L_{\odot}$ to $10^{-4} L_{\odot}$. We use a definition of habitability based on the presence of surface liquid water, and find that most of the planets considered can sustain surface liquid water on the dayside with an ice cap on the nightside. However, for high eccentricity and high luminosity, planets cannot sustain surface liquid water during the whole orbital period. They completely freeze at apoastron and when approaching periastron an ocean appears around the substellar point. We conclude that the higher the eccentricity and the higher the luminosity of the star, the less reliable the mean flux approximation.
\end{abstract}

Key words. planets and satellites: atmospheres - planets and satellites: terrestrial planets - methods: numerical

\section{Introduction}

The majority of the planets found in the insolation habitable zone (HZ, the zone in which a planet could sustain surface liquid water, as defined by Kasting et al. 1993) are on eccentric orbits. The actual percentage depends on the definition of the inner and outer edges considered for the HZ. For instance, about $80 \%$ of the planets spending some time in the conservative HZ, whose the inner edge corresponds to the "runaway greenhouse" criterium and the outer edge to the "maximum greenhouse" criterium (e.g., Kopparapu 2013; Kopparapu et al. 2014) have an eccentricity of more than $0.1^{1}$.

While most of the planets detected in the HZ are very massive planets and are probably gaseous, five of them have masses below $10 M_{\oplus}$ and eleven of them have radii smaller than $2 R_{\oplus}$ (such as Kepler-186f with an estimated eccentricity of $\sim 0.01$; Quintana et al. 2014). Among these sixteen possibly rocky planets, four of them have eccentricities higher than 0.1: GJ 832 c (Bailey et al. 2009), Kepler-62 e, Kepler-69 c (Borucki et al. 2011), and GJ 667 Cc (Anglada-Escudé et al. 2012; Robertson \& Mahadevan 2014). Table 1 shows the

\footnotetext{
1 http://physics.sfsu. edu/ skane/hzgallery/index.html
}

characteristics of these four planets and the percentage of the orbital phase spent within the HZ for two different definitions of the inner and outer edges.

We expect more small planets to be discovered in the $\mathrm{HZ}$ with the future missions to increase statistics (e.g., NGTS, TESS) and also better constrain eccentricity (e.g., PLATO). In any case, the discovery of the 4 planets mentioned above raise the question of the potential habitability of planets, like GJ $832 \mathrm{c}$ and GJ $667 \mathrm{Cc}$, that only spend a fraction of their orbit in the HZ.

The influence of the orbital eccentricity of a planet on its climate has already been studied using various methods: energybalanced models (EBMs) and global climate models (GCMs). EBMs assume that the planet is in thermal equilibrium: on average, the planet must radiate the same amount of long-wave radiation to space as the short-wave radiation it receives from the host star (Williams \& Kasting 1997). In EBM models, the radiative energy fluxes entering or leaving a cell are balanced by the dynamic fluxes of heat transported by winds into or away from the cell. On the contrary, GCMs consistently compute on a three-dimensional grid the circulation of the atmosphere using forms of the Navier-Stokes equations. GCMs are therefore more 
Table 1. Possibly rocky observed exoplanets with an eccentricity higher than 0.1 (from the Habitable Zone Gallery, Kane \& Gelino 2012).

\begin{tabular}{|c|c|c|c|c|c|c|c|c|c|c|}
\hline Planets & $\begin{array}{c}M_{\star} \\
\left(M_{\odot}\right)\end{array}$ & $\begin{array}{l}T_{\text {eff }} \\
(\mathrm{K})\end{array}$ & $\begin{array}{c}M_{\mathrm{p}} \\
\left(M_{\oplus}\right)\end{array}$ & $\begin{array}{c}R_{\mathrm{p}} \\
\left(R_{\oplus}\right) \\
\end{array}$ & $\begin{array}{c}\text { Periastron-apoastron } \\
(\mathrm{au})\end{array}$ & Eccentricity & $\begin{array}{l}\mathrm{HZ}_{\text {in-out,c }} \\
\quad(\mathrm{au})\end{array}$ & $\begin{array}{l}\mathrm{HZ}_{\text {in-out,o }} \\
\quad(\mathrm{au})\end{array}$ & $\begin{array}{c}\tau_{\mathrm{HZ}, \mathrm{c}} \\
(\%)\end{array}$ & $\begin{array}{c}\tau_{\mathrm{HZ}, \mathrm{o}} \\
(\%)\end{array}$ \\
\hline Kepler-62 e & 0.69 & 4925 & & 1.6 & $0.37-0.49$ & 0.13 & $0.46-0.84$ & $0.37-0.89$ & 29.9 & 100 \\
\hline Kepler-69 c & 0.81 & 5640 & & 1.7 & $0.61-0.81$ & 0.14 & $0.85-1.50$ & $0.67-1.59$ & 0 & 66.9 \\
\hline GJ 832 c & 0.45 & 3500 & 5.4 & & $0.13-0.19$ & 0.18 & $0.19-0.36$ & $0.15-0.38$ & 24.7 & 73.3 \\
\hline GJ $667 \mathrm{Cc}$ & 0.33 & 3350 & 3.8 & & $0.09-0.16$ & 0.27 & $0.15-0.29$ & $0.12-0.31$ & 29.5 & 35.2 \\
\hline
\end{tabular}

Notes. $\mathrm{HZ}_{\text {in-out,c }}$ corresponds to the inner and outer edge of the conservative $\mathrm{HZ}$ and $\mathrm{HZ}_{\text {in-out,o }}$ corresponds to the inner and outer edge of the optimistic HZ (the inner edge corresponds to the "recent Venus" criterium and the outer edge to the "early Mars" criterium; e.g., Kopparapu 2013; Kopparapu et al. 2014). $\tau_{\mathrm{HZ}, \mathrm{c}}$ is the percentage of the orbital phase spent within the conservative $\mathrm{HZ}$ and $\tau_{\mathrm{HZ}, \mathrm{o}}$ is the percentage of the orbital phase spent within the optimistic HZ.

computationally demanding, but they are more accurate when simulating a climate.

Using a GCM, Williams \& Pollard (2002) studied the influence of the eccentricity on the climate of Earth-like planets around a Sun-like star (with the correct distribution of continents and oceans, a 365 day orbit, a $24 \mathrm{~h}$ day, and a $23^{\circ}$ obliquity) and found that surface liquid water is possible even on very eccentric orbits. Using a GCM, Linsenmeier et al. (2015) studied the influence of both obliquity and eccentricity for ocean covered planets orbiting a Sun-like star on a 365 day orbit and a 24 hour day, like Earth. They found that planets with eccentricities higher than 0.2 can only sustain surface liquid water for a part of the year.

Spiegel et al. (2010) and Dressing et al. (2010) used EBMs to illustrate the effect of the evolution of eccentricity (through pseudo-Milankovitch's cycles; Milankovitch 1941). Spiegel et al. (2010) found that the increase of eccentricity of a planet may allow it to escape a frozen snowball state. Dressing et al. (2010) found that increasing the eccentricity widens the parameter space in which the planet can only sustain surface liquid water for part of the year.

A major result of Williams \& Pollard (2002) was that the capacity of an eccentric planet of semi-major axis $a$ and eccentricity $e$ to host surface liquid water depends on the average flux received over one orbit. This average flux corresponds to the flux received by a planet on a circular orbit of radius $r=a\left(1-e^{2}\right)^{1 / 4}$. If this orbital distance is within the HZ, then the planet is assumed to belong to the HZ (or to the eccentric HZ, as defined by Barnes et al. 2008). However this study was performed by simulating the climate of an Earth-twin planet. The generalization of this result to the diversity of the planets discovered in the HZ is not straightforward. We expect this mean-flux approximation to be adequate for planets with low eccentricities; however, for high eccentricities the climate could be drastically degraded when the planet is temporarily outside the HZ. This would especially be an issue for planets around hot stars where the HZ is far from the star. The planet could spend a long time outside of the HZ, leading to the freezing of the water reservoir at apoastron and its evaporation at periastron.

The influence of the stellar luminosity/host star type has previously been considered for Earth-like planets on circular orbits (e.g., Shields et al. 2013, 2014) and Wordsworth et al. (2011) have studied the climate of GJ 581d orbiting a red dwarf for two different eccentricities ( 0 and 0.38 ), but no work has studied jointly the influence of the planet's eccentricity and the stellar luminosity.

We therefore aimed to explore here, in a systematic way, the influence of the planet's eccentricity and the star luminosity on the climate of ocean covered planets in a 1:1 spin-orbit resonance, that receive on average the same flux as Earth. In order to test the limits of the mean flux approximation, we performed three-dimensional GCM simulations for a wide range of configurations: we considered stars of luminosity $1 L_{\odot}, 10^{-2} L_{\odot}$ and $10^{-4} L_{\odot}$ and orbits of eccentricity from 0 to 0.9 . We took into account the different luminosities by scaling the orbital periods of the planets. This means that we did not consider here the spectral dependance of the stars. We investigated whether these planets were able to sustain surface liquid water.

In Sect. 2 we present our definition of habitability in terms of surface liquid water coverage. In Sect. 3 we explain the set-up of our simulations, and in Sects. 4 and 5 we discuss their outcome in terms of liquid water coverage. In Sect. 7 we discuss the observability of the variability caused by eccentricity. Finally, in Sects. 8 and 9 we conclude this study.

\section{Liquid water coverage vs. habitability}

We do not consider that habitability is equivalent to the requirement of having a mean surface temperature higher than the freezing point of water, as do the energy balance models (e.g., Williams \& Kasting 1996) or the radiative-convective models (e.g., Kasting et al. 1993). As in Spiegel et al. (2008), we chose an assessment of the habitability of a planet based on sea ice cover. We focus here only on the presence of surface liquid water and not on the actual potential of the planets to be appropriate environments for the appearance of life.

The planets considered in this work are water worlds (or aqua worlds), i.e., planets whose whole surface is covered with water (here treated as an infinite water source). Considering water worlds is especially convenient for a first study because it allows us to have a small number of free parameters (no land/ocean distribution, land roughness, etc.). A subset of this population are the ocean planets with a high bulk water fraction, which strongly alters their internal structure. Ocean planets were hypothesized in the early 2000s by Kuchner (2003) and Léger et al. (2004). They are believed to have a mass ranging from $1 M_{\oplus}$ (small rocky planets) to $10 M_{\oplus}$ (mini-Neptunes). Their composition was investigated, and the depth of the ocean of a Earth-mass planet was estimated to a few hundred kilometers (Sotin et al. 2007). These planets could be identified providing that we knew the mass and radius with enough precision (Sotin et al. 2007; Selsis et al. 2007). Despite the lack of knowledge of their mass, some observed planets have been proposed to be ocean planets, for example Kepler-62e and $-62 \mathrm{f}$ (Kaltenegger et al. 2013). 


\section{GCM simulations}

\subsection{Model parameters}

We performed the climate simulations with the $\mathrm{LMDZ}^{2}$ generic global climate model (GCM) widely used for the study of extrasolar planets (e.g., Wordsworth et al. 2010, 2011; Selsis et al. 2011) and the paleoclimates of Mars (Wordsworth et al. 2013; Forget et al. 2013). In particular, we used the three-dimensional dynamical core of the LMDZ 3 GCM (Hourdin et al. 2006), based on a finite-difference formulation of the primitive equations of geophysical fluid dynamics. A spatial resolution of $64 \times 48 \times 30$ in longitude, latitude, and altitude was set for the simulations.

We assumed that the atmosphere is mainly composed of $\mathrm{N}_{2}$, with $376 \mathrm{ppmv}^{3}$ of $\mathrm{CO}_{2}$, which corresponds to an Earth-like atmosphere. The water cycle was modeled with a variable amount of water vapor and ice. Ice formation (melting) was assumed to occur when the surface temperature is lower (higher) than $273 \mathrm{~K}$, and temperature changes due to the latent heat of fusion were taken into account.

We used the high-resolution spectra computed by Leconte et al. (2013) over a range of temperatures and pressures using the HITRAN 2008 database (Rothman et al. 2009). We adopted the same temperature grids as in Leconte et al. (2013) with values $T=\{110,170, \ldots, 710\} \mathrm{K}$ and the same pressure grids with values $p=\left\{10^{-3}, 10^{-2}, \ldots, 10^{5}\right\}$ mbar. The water volume mixing ratio could vary between $10^{-7}$ to 1 . The $\mathrm{H}_{2} \mathrm{O}$ absorption lines were truncated at $25 \mathrm{~cm}^{-1}$, but the water vapor continuum was included using the CKD model (Clough et al. 1989). As in Leconte et al. (2013), the opacity due to $\mathrm{N}_{2}-\mathrm{N}_{2}$ collision-induced absorption was also taken into account.

We used the same correlated-k method as in Wordsworth et al. (2011) and Leconte et al. (2013) to produce a smaller database of spectral coefficients suitable for fast calculation of the radiative transfer in the GCM. The model used 38 spectral bands for the thermal emission of the planet and 36 for the stellar emission. In our water world model, we did not take into account the oceanic circulation. We chose an albedo of 0.07 for the surface liquid water and an albedo of 0.55 for the ice and snow.

We considered the influence of some parameters on the outcome of our simulations: the thermal inertia $I_{\mathrm{oc}}$ of the oceans and the maximum ice thickness $h_{\text {ice }}$ allowed in our model. We tested three combinations:

$\left\{\begin{array}{ll}h_{\text {ice }}=1 \mathrm{~m}, & I_{\mathrm{oc}}=18000 \mathrm{~J} \mathrm{~s}^{-1 / 2} \mathrm{~m}^{-2} \mathrm{~K}^{-1}, \\ h_{\text {ice }}=10 \mathrm{~m}, & I_{\mathrm{oc}}=18000 \mathrm{~J} \mathrm{~s}^{-1 / 2} \mathrm{~m}^{-2} \mathrm{~K}^{-1} \\ h_{\text {ice }}=1 \mathrm{~m}, & I_{\mathrm{oc}}=36000 \mathrm{~J} \mathrm{~s}^{-1 / 2} \mathrm{~m}^{-2} \mathrm{~K}^{-1}\end{array}\right.$,

The results given in Sects. 4, 5 were obtained with the first combination, while comparisons with the other two sets of values is performed in Sect. 6. Changing thermal inertia is a way to model the ocean mixed layer depth, which responds quickly to the climatic forcing. This mixed layer varies on Earth with location and time. Selsis et al. (2013) studied the effect of changing the thermal inertia of hot planets without atmospheres. They showed that increasing the thermal inertia of the surface of such a planet damped the amplitude of its temperature response to the eccentricity-driven insolation variations. They also showed that increasing thermal inertia introduced a lag in the response of

\footnotetext{
2 Model developed at the Laboratoire de Météorologie Dynamique, the Z of LMDZ standing for Zoom capability.

3 Parts per million by volume.
}

the planet with respect to the insolation variations. Imposing the maximum ice thickness allowed in the model is a way to mimic oceanic transports that limit the growth of ice layers. It influences the time it takes to reach equilibrium and the eccentricitydriven oscillations (see Sect. 6).

\subsection{Planets and initial conditions}

We computed the climate of water worlds, initially ice-free, and obtained a mean flux equal to Earth's $\left(1366 \mathrm{~W} / \mathrm{m}^{2}\right)$ on orbits of eccentricity from 0 to 0.9 around stars of different luminosities:

- $L_{\star}=1 L_{\odot}$, corresponding to our Sun with an effective temperature of $\sim 5800 \mathrm{~K}$;

- $L_{\star}=10^{-2} L_{\odot}$, corresponding to a M-dwarf of $0.25 M_{\odot}$ with an effective temperature of $\sim 3300 \mathrm{~K}$;

- $L_{\star}=10^{-4} L_{\odot}$, corresponding to a 500 Myr brown-dwarf of mass $0.04 M_{\odot}$ with an effective temperature of $\sim 2600 \mathrm{~K}$.

We note that we did not take into account in this work the spectral dependance of the stars. For instance, we did not consider that a $10^{-4} L_{\odot}$ star is much redder than a $1 L_{\odot}$ star. We took into account the different luminosities only by scaling the orbital period of the planets, as explained in the following.

We considered here planets in a 1:1 spin-orbit resonance, regardless of their eccentricity. However, a planet orbiting a $1 L_{\odot}$ star on a circular orbit and receiving a flux of $1366 \mathrm{~W} / \mathrm{m}^{2}$ (i.e., at an orbital distance of 1 au) will not reach a synchronous rotation state in less than the age of the Universe. Moreover, if the planet is very eccentric, the probability that it is in synchronous rotation is low. The planet will more likely be either in pseudosynchronization (synchronization at periastron; Hut 1981) or in spin-orbit resonance (Makarov \& Efroimsky 2013). The aim of our work was to investigate the effect of eccentricity and luminosity, so we only varied here these two parameters and kept all the others equal. Choosing a synchronous rotation allowed us to have, for a given eccentricity, the exact same insolation evolution for planets orbiting a high luminosity star as a low luminosity star. The obliquity of the planet was assumed to be zero. In all cases, the simulations were run from an initial ice-free state until runaway greenhouse/glaciation occurred or steady states of thermal equilibrium were reached.

For the different eccentricities, we scaled the orbital period of the planet (the duration of the "year") to insure that the planet receives $F_{\oplus}=1366 \mathrm{~W} / \mathrm{m}^{2}$ on average. A planet of semi-major axis $a$ and eccentricity $e$ receives an averaged flux over one orbit of

$F=\frac{L_{\star}}{4 \pi a^{2} \sqrt{1-e^{2}}}$

where $L_{\star}$ is the luminosity of the star. In our study, we assume that the planet receives on average $F_{\oplus}$,

$F=F_{\oplus}=\frac{L_{\odot}}{4 \pi a_{\oplus}^{2}}=1366 \mathrm{~W} / \mathrm{m}^{2}$

where $a_{\oplus}=1$ au. Thus, we can express the semi-major axis of the planet as a function of eccentricity $e$ and stellar luminosity $L_{\star}$ :

$a=\frac{a_{\oplus}}{\left(1-e^{2}\right)^{1 / 4}} \sqrt{\frac{L_{\star}}{L_{\odot}}}$.

If we increase the eccentricity of the orbit of the planet, its semimajor axis $a$ increases to ensure it receives on average $F_{\oplus}$. For 
Table 2. Planets' orbit characteristics and received flux for $L_{\star}=L_{\odot}$.

\begin{tabular}{crrrrrr}
\hline \hline & \multicolumn{5}{c}{$L_{\star}=L_{\odot}$} \\
\cline { 2 - 7 } Ecc & $a(\mathrm{au})$ & Peri $(\mathrm{au})$ & Apo $(\mathrm{au})$ & $P_{\text {orb }}($ day $)$ & Flux at peri $\left(\mathrm{W} / \mathrm{m}^{2}\right)$ & Flux at apo $\left(\mathrm{W} / \mathrm{m}^{2}\right)$ \\
\hline 0 & 1.000 & 1.00 & 1.00 & 365.5 & 1366 & 1366 \\
0.05 & 1.001 & 0.95 & 1.05 & 365.9 & 1517 & 1241 \\
0.1 & 1.003 & 0.90 & 1.10 & 366.9 & 1697 & 1136 \\
0.2 & 1.011 & 0.81 & 1.21 & 371.2 & 2128 & 954 \\
0.4 & 1.045 & 0.63 & 1.46 & 390.2 & 3758 & 700 \\
0.6 & 1.119 & 0.45 & 1.79 & 432.1 & 8447 & 534 \\
0.8 & 1.292 & 0.26 & 2.33 & 536.2 & 33731 & 420 \\
0.9 & 1.516 & 0.15 & 2.88 & 681.4 & 139530 & 378 \\
\hline
\end{tabular}

Notes. $a$ is the semi-major axis defined in Eq. (3). Because the planets are in synchronous rotation, the orbital period $\left(P_{\text {orb }}\right.$ columns, given in Earth days $=24 \mathrm{~h}$ ) and the rotation period of the planets are equal. Peri denotes the periastron distance and apo the apoastron distance.

Table 3. Planets' orbit characteristics for $L_{\star}=10^{-2} L_{\odot}$ and $L_{\star}=10^{-4} L_{\odot}$.

\begin{tabular}{|c|c|c|c|c|c|c|c|c|}
\hline \multirow[b]{2}{*}{ Ecc } & \multicolumn{4}{|c|}{$L_{\star}=10^{-2} L_{\odot}$} & \multicolumn{4}{|c|}{$L_{\star}=10^{-4} L_{\odot}$} \\
\hline & $a(\mathrm{au})$ & Peri (au) & Apo $(\mathrm{au})$ & $P_{\text {orb }}$ (day) & $a(\mathrm{au})$ & Peri (au) & Apo $(\mathrm{au})$ & $P_{\text {orb }}($ day $)$ \\
\hline 0 & $1.000 \times 10^{-1}$ & $1.00 \times 10^{-1}$ & $1.00 \times 10^{-1}$ & 22.85 & $1.000 \times 10^{-2}$ & $1.00 \times 10^{-2}$ & $1.00 \times 10^{-2}$ & 1.967 \\
\hline 0.05 & $1.001 \times 10^{-1}$ & $0.95 \times 10^{-1}$ & $1.05 \times 10^{-1}$ & 22.87 & $1.001 \times 10^{-2}$ & $0.95 \times 10^{-2}$ & $1.05 \times 10^{-2}$ & 1.968 \\
\hline 0.1 & $1.003 \times 10^{-1}$ & $0.90 \times 10^{-1}$ & $1.10 \times 10^{-1}$ & 22.94 & $1.003 \times 10^{-2}$ & $0.90 \times 10^{-2}$ & $1.10 \times 10^{-2}$ & 1.974 \\
\hline 0.2 & $1.011 \times 10^{-1}$ & $0.81 \times 10^{-1}$ & $1.21 \times 10^{-1}$ & 23.21 & $1.011 \times 10^{-2}$ & $0.81 \times 10^{-2}$ & $1.21 \times 10^{-2}$ & 1.997 \\
\hline 0.4 & $1.045 \times 10^{-1}$ & $0.63 \times 10^{-1}$ & $1.46 \times 10^{-1}$ & 24.40 & $1.045 \times 10^{-2}$ & $0.63 \times 10^{-2}$ & $1.46 \times 10^{-2}$ & 2.099 \\
\hline 0.6 & $1.119 \times 10^{-1}$ & $0.45 \times 10^{-1}$ & $1.79 \times 10^{-1}$ & 27.02 & $1.119 \times 10^{-2}$ & $0.45 \times 10^{-2}$ & $1.79 \times 10^{-2}$ & 2.325 \\
\hline 0.8 & $1.292 \times 10^{-1}$ & $0.26 \times 10^{-1}$ & $2.33 \times 10^{-1}$ & 33.52 & $1.292 \times 10^{-2}$ & $0.26 \times 10^{-2}$ & $2.33 \times 10^{-2}$ & 2.885 \\
\hline 0.9 & $1.516 \times 10^{-1}$ & $0.15 \times 10^{-1}$ & $2.88 \times 10^{-1}$ & 42.60 & $1.516 \times 10^{-2}$ & $0.15 \times 10^{-2}$ & $2.88 \times 10^{-2}$ & 3.666 \\
\hline
\end{tabular}

Notes. The fluxes at periastron and apoastron are the same as in Table 2.

example, a planet around a Sun-like star with an eccentricity of 0.6 and receiving $1366 \mathrm{~W} / \mathrm{m}^{2}$ on average has a semi-major axis of 1.119 au.

Table 2 shows the different values of the semi-major axis of the planets for different eccentricities, as well as the distances of periastron and apoastron, and the fluxes the planet receives at these distances for $L_{\star}=L_{\odot}$. Table 3 shows the planets' orbit characteristics for $L_{\star}=10^{-2} L_{\odot}$ and $L_{\star}=10^{-4} L_{\odot}$. Since we consider synchronous planets, we can deduce the rotation period of the planets depending on the eccentricity and the type of the star. For a star of $L_{\star}=1 L_{\odot}$ and a planet on a circular orbit, the year is 365 days long and the planet has a slow rotation. If the planet is on a very eccentric orbit $(e=0.9)$, then the semimajor axis is $1.516 \mathrm{au}$, the year is 681 days long, and the planet has an even slower rotation. For a star of $L_{\star}=10^{-4} L_{\odot}$ and a planet on a circular orbit, the semi-major is $0.01 \mathrm{au}$, the year is $\sim 2$ days long ( $\sim 4$ days for $e=0.9)$, and the planet has a faster rotation.

In addition, as shown in Selsis et al. (2013), because of the optical libration due to the 1:1 spin-orbit resonance, there is no permanent dark area on a planet with an eccentricity higher than 0.72. We define here the dayside as the hemisphere that is illuminated when the planet has an eccentricity of 0 . For this case, the substellar point is at $0^{\circ}$ longitude and $0^{\circ}$ latitude, and the dayside extends to $-90^{\circ}$ to $90^{\circ}$ in longitude and $-90^{\circ}$ to $90^{\circ}$ in latitude. The nightside is the other side of the planet, i.e., from $90^{\circ}$ to $270^{\circ}$ in longitude and $-90^{\circ}$ to $90^{\circ}$ in latitude. We use this geographic definition of the dayside and nightside independently of the eccentricity of the orbit.
We first discuss the effect of varying the star luminosity on the climate of planets on circular orbits (Sect. 4), then we extend the discussion to planets on eccentric orbits (Sect. 5).

\section{Circular orbits}

In Sect. 4.1, we first give our results for a star of luminosity $L_{\star}=1 L_{\odot}$. In Sect. 4.2, we compare them with those obtained for a star of luminosity $L_{\star}=10^{-2} L_{\odot}$ and $L_{\star}=10^{-4} L_{\odot}$.

\section{1. $L_{\star}=1 L_{\odot}$}

Figure 1 shows the longitude-latitude maps of surface temperature, ice density, water vapor, and precipitation on a planet orbiting a Sun-like star after 10000 days. The mean surface temperature needs about $\sim 1500$ days to reach its equilibrium, which is about $267 \mathrm{~K}$. As the planet is in synchronous rotation, the dayside is much hotter than the nightside (Fig. 1, top left). The temperatures on the dayside reach $320 \mathrm{~K}$ at the substellar point, whereas temperatures on the nightside are around $240 \mathrm{~K}$. From an initially free water ocean, an ice cap forms in a few hundred years (Fig. 1, top right). About $62 \%$ of the planet is covered with ice and $38 \%$ of the ocean remains free of ice around the substellar point. We obtain the same kind of features as an eyeball planet (like in Pierrehumbert 2011 and Wordsworth et al. 2011 for GJ 581d). Evaporation occurs on a ring around the substellar point (Fig. 1, bottom right), and there is a lot of precipitation at the substellar point owing to humidity convergence 
Surface temperature

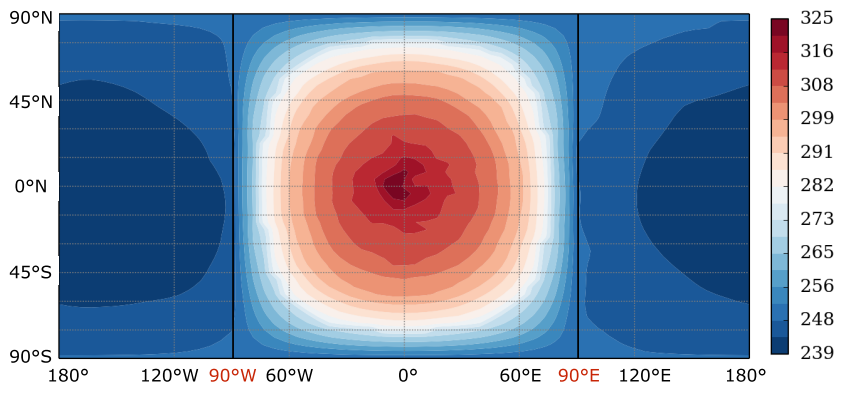

Water vapor

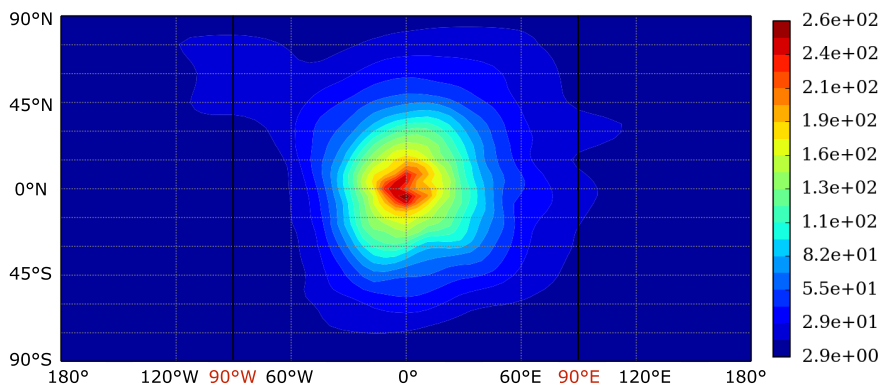

Surface ice density

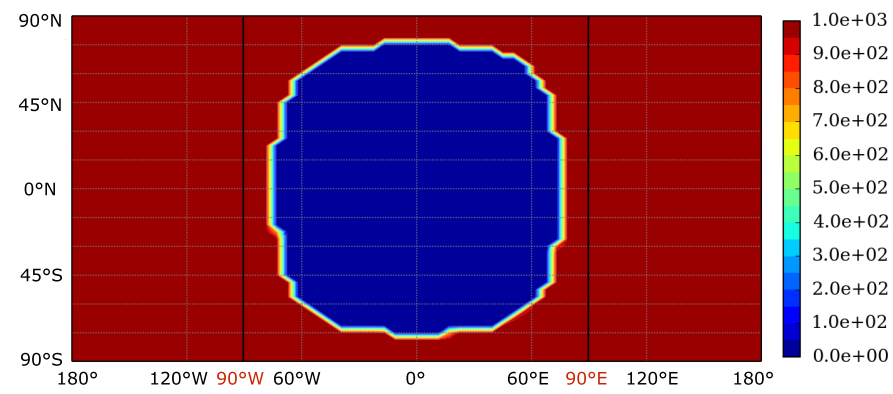

Surface water variations

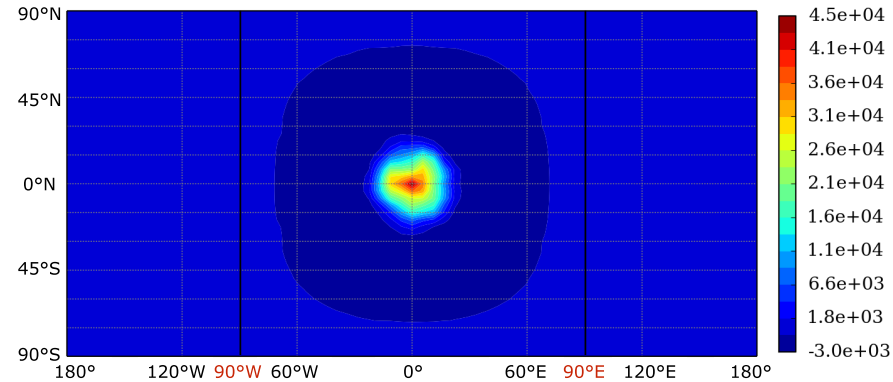

Fig. 1. Maps of surface temperature, ice density, amount of water vapor integrated on a column, and precipitation for a planet around a $1 L_{\odot}$ star on a circular orbit. The terminator (the longitudes of $90^{\circ} \mathrm{W}$ and $90^{\circ} \mathrm{E}$ ) is shown in black. For the map showing the surface water variation, a negative value means that liquid water disappears (evaporation or freezing) and a positive value means that liquid water appears (rain or melting). For the map showing the surface ice density, a density of $1000 \mathrm{~kg} / \mathrm{m}^{2}$ corresponds to a $1000 \mathrm{~mm}=1 \mathrm{~m}$ thick ice layer.

and condensation of moisture along the ascending branch of an Hadley type cell (Fig. 1, bottom left).

The albedo of the planet is about 0.25 , which is significantly lower than in Yang et al. (2013). This might be due to several differences in our simulations. For example, the albedo depends on the size of the cloud-forming ice grains. The bigger they are, the lower the albedo. In our model, the cloud ice particles have a size that varies depending on the water mixing ratio (see Leconte et al. 2013 for details). In Yang et al. (2013) the size is not indicated, but is said to be the same size as observed on Earth. Furthermore, Yang et al. (2013) pointed out that the albedo of the planet strongly depends on the oceanic transport, which is not included here. Finally, the biggest difference comes from the moist convection parametrization, which is chosen to be very simple with few free parameters in LMDZ (Manabe \& Wetherald 1967). This parametrization leads to a lower cloud cover, and thus a lower albedo.

Figure 2 shows the dayside and nightside mean profiles of temperature, water ice, and water vapor in the atmosphere. These mean profiles were obtained by performing a time average over one orbital period ${ }^{4}$. The surface dayside temperatures are higher than the surface nightside temperatures. Thanks to this lower surface temperature, we can see a temperature inversion on the nightside, which occurs at a pressure level of about 1 bar. On the dayside, water ice clouds are located around an altitude of $\sim 15 \mathrm{~km}$ ( $\sim 90 \mathrm{mbar})$, while on the nightside, there are no water ice clouds in the atmosphere. The concentration of water vapor is much higher on the dayside than on the nightside. For both the dayside and nightside, the water vapor in the atmosphere is

454 points per orbit were used for the case $1 L_{\odot}\left(P_{\text {orb }}=365.5\right.$ day $)$, 122 points for $10^{-2} L_{\odot}\left(P_{\text {orb }}=22.85\right.$ day $)$, and 98 points for $10^{-4} L_{\odot}$ $\left(P_{\text {orb }}=1.967\right.$ day $)$. The number of points depends on the output time frequency. essentially found within the first $20 \mathrm{~km}$ of the atmosphere (pressure $>0.1$ bar)

On average, the concentration of water vapor in the dayside upper atmosphere (pressure $<10$ mbar) is about $1 \times 10^{-9} \mathrm{~kg} / \mathrm{m}^{2}$, which is about 100 times more than the water vapor concentration on Earth at the same altitude (Butcher et al. 1992). It is therefore possible that these planets experience little water escape, as on Earth (Lammer et al. 2003; Kulikov et al. 2007; Selsis et al. 2007). We note that an extreme case for water escape has been observed for a hot Neptune (Ehrenreich et al. 2015). However, we expect a much lower escape rate here as the planet is located much farther away. There are ice clouds above the substellar point at an altitude of $15 \mathrm{~km}$; these clouds protect the substellar point. Yang et al. (2013) determined that this mechanism allows the inner edge of the insolation $\mathrm{HZ}$ for synchronous planets to be closer than for non-synchronous planets.

In conclusion, during its orbit a synchronized planet on a circular orbit around a $1 L_{\odot}$ star always has a part of its ocean ice-free on the dayside (around the substellar point) and covered by an ice cap on its nightside.

\subsection{Decreasing the luminosity}

Decreasing the stellar luminosity ${ }^{5}$ changes the global characteristics of the planet's climate, such as the surface temperature map and the surface ice density. The lower the luminosity the bigger the differences with the previous case.

5 We do not take into account the spectral dependance of a low luminosity star. Decreasing the luminosity is done in our work by decreasing the orbital period of the planet, and thus its rotation period (Tables 2 and 3). 
a) Dayside
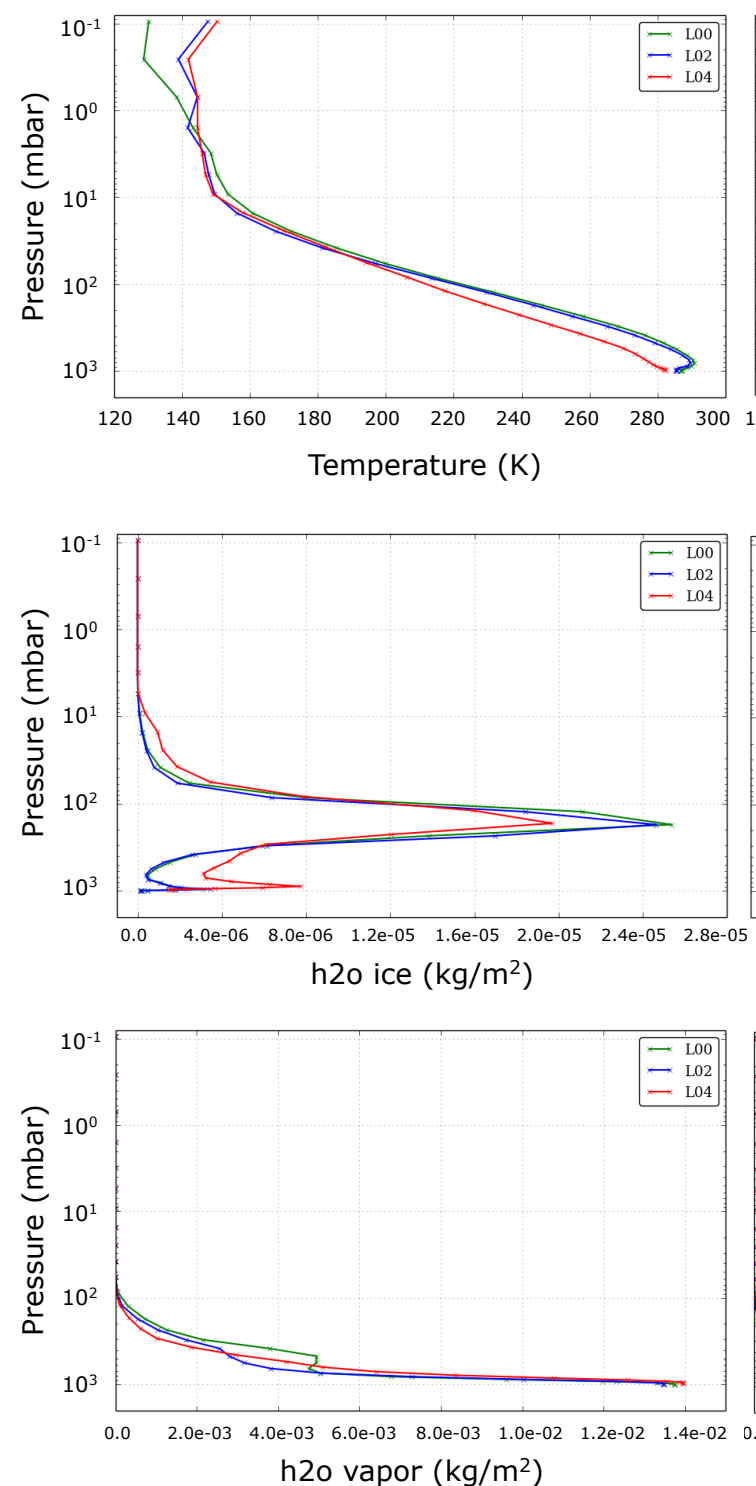

b) Nightside
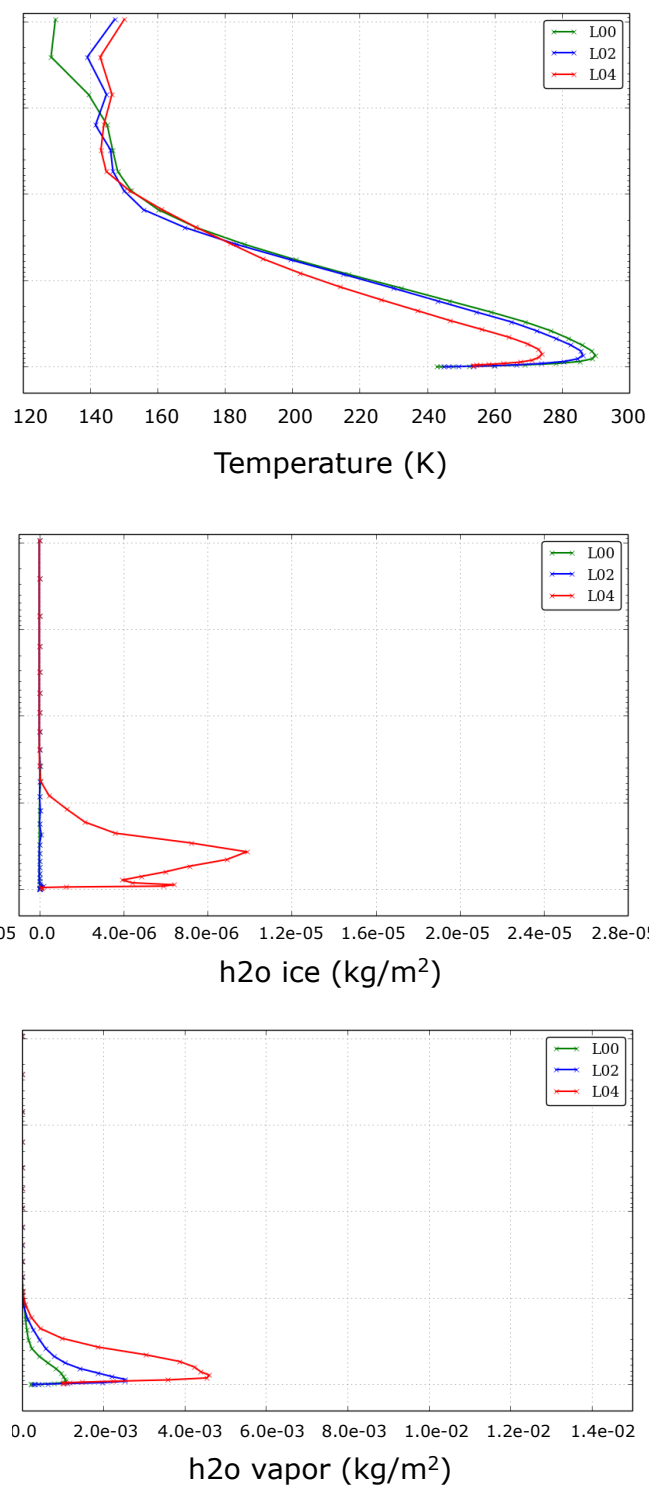

Fig. 2. a) Dayside and b) nightside mean profiles of temperature (top panels), water ice (middle panels), and water vapor (bottom panels) in the atmosphere of a planet on a circular orbit around a $1 L_{\odot}$ star (L00, in green), a $10^{-2} L_{\odot}$ star (L02, in blue), and a $10^{-4} L_{\odot}$ star (L04, in red).

\subsection{1. $L_{\star}=10^{-2} L_{\odot}$}

Figure 2 shows that there is little difference between the mean temperature profiles at $1 L_{\odot}$ and $10^{-2} L_{\odot}$. The temperature at low altitudes (pressure $>2$ mbar) is slightly lower, but in the upper atmosphere (pressure $<2$ mbar) it is higher (the difference reaches 10 to $20 \mathrm{~K}$ ). The water ice clouds follow the same distribution as for a $1 L_{\odot}$ star, with a high density at an altitude of $\sim 15 \mathrm{~km}$ ( $\sim 90 \mathrm{mbar})$ on the dayside and very few clouds on the nightside. The water vapor distribution is very similar between the luminosities $1 L_{\odot}$ and $10^{-2} L_{\odot}$ and the concentration becomes negligible above an altitude of $\sim 20 \mathrm{~km}$ (pressure $<100 \mathrm{mbar}$ ).

However, owing to the faster rotation, the temperature distribution is different. This is due to the higher Coriolis force that strengthens the mechanisms responsible for the equatorial super-rotation. The wave-mean flow interaction identified by Showman \& Polvani (2011) and the three-way force balance identified by Showman et al. (2013, 2015) both affect the atmospheric circulation (see also Leconte et al. 2013, for a discussion of the transition from stellar-antistellar circulation to super-rotation in the specific context of terrestrial planets). Figure 3 shows maps of the atmospheric temperature (color maps) and the wind pattern (with vectors) at an altitude of $10 \mathrm{~km}$ (corresponding to a pressure of 305 mbar for $1 L_{\odot}, 301 \mathrm{mbar}$ for $10^{-2} L_{\odot}$, and $190 \mathrm{mbar}$ for $\left.10^{-4} L_{\odot}\right)$. For $1 L_{\odot}$, the wind is weak and isotropically transports heat away from the substellar point (stellar/anti-stellar circulation, as shown in Leconte et al. 2013 , for a slowly rotating Gl-581c). However, for $10^{-2} L_{\odot}$ the wind is stronger, especially the longitudinal component, and redistributes the heat more efficiently toward the east. Thanks to this stronger wind, there is an asymmetry of the surface temperature distribution on the planet, so that the east is hotter than the west and the temperature is more homogeneous along the equator. The planet orbiting a $10^{-2} L_{\odot}$ star is colder at the poles (surface temperature of $\sim 235 \mathrm{~K}$ ) than for $1 L_{\odot}$ star (surface temperature of $\sim 250 \mathrm{~K}$ ). The wind pattern here is marked by the presence of a Rossby wave typical of the Rossby wave transition region (as defined in Carone et al. 2015, for tidally locked 

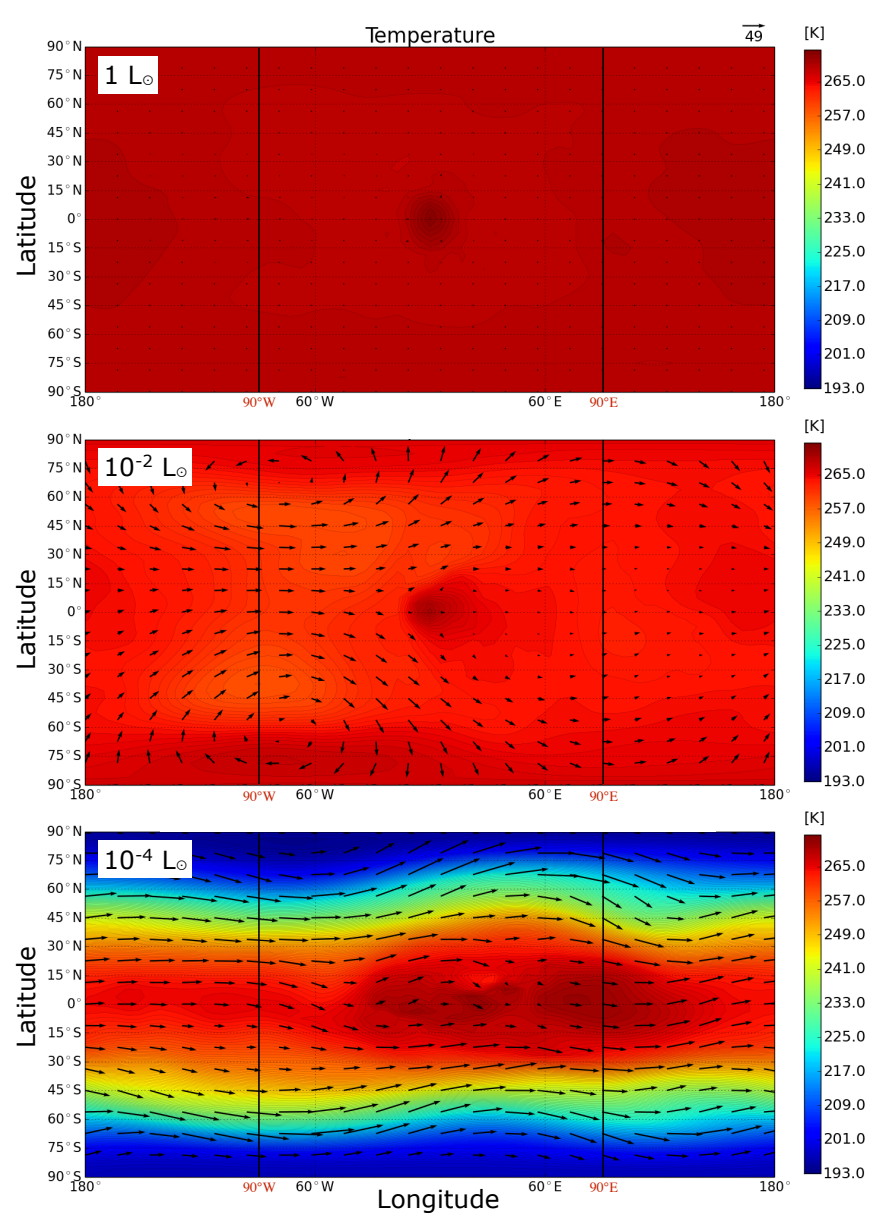

Fig. 3. Maps of the atmospheric temperature (color map) and winds (vectors are in units of $\mathrm{m} / \mathrm{s}$, legend at the top of the graph) at an altitude of $10 \mathrm{~km}$ for a planet on a circular orbit around a $1 L_{\odot}$ star (top), a $10^{-2} L_{\odot} \operatorname{star}$ (middle), and a $10^{-4} L_{\odot} \operatorname{star}($ bottom).

planets). In Carone et al. (2015), the Rossby wave transition region for an Earth-size planet is said to occur for a rotation period of 25 days, which is approximately the rotation period here (22.85 days). In the $1 L_{\odot}$ case, the rotation period was much longer and no Rossby wave could develop (e.g., Leconte et al. 2013).

Moreover, for $10^{-2} L_{\odot}$, the average ice density is similar to the value of the planet orbiting a $1 L_{\odot}$ star. However, owing to the asymmetric surface temperature due to the Coriolis force, the shape of the ice-free region is slightly different. Figure 4 shows the shape of the surface ice density for $L_{\star}=10^{-2} L_{\odot}$ (top panel). Although the percentage of the ice-free region remains the same, the ocean for $10^{-2} L_{\odot}$ reaches slightly lower latitudes and is more extended longitudinally than for $1 L_{\odot}$.

Despite these small changes with respect to the case $1 L_{\odot}$, a planet on a circular orbit around a $10^{-2} L_{\odot}$ star is therefore equally capable of sustaining surface liquid water.

\subsection{2. $L_{\star}=10^{-4} L_{\odot}$}

For an even less luminous host body, the differences that appeared for the case $L_{\star}=10^{-2} L_{\odot}$ are accentuated. The temperature maps and surface ice density maps show a more longitudinal extension than before. Owing to the even higher rotation rate of the planet (rotation period of 2 days, see Table 3), the winds redistribute heat longitudinally from the substellar point
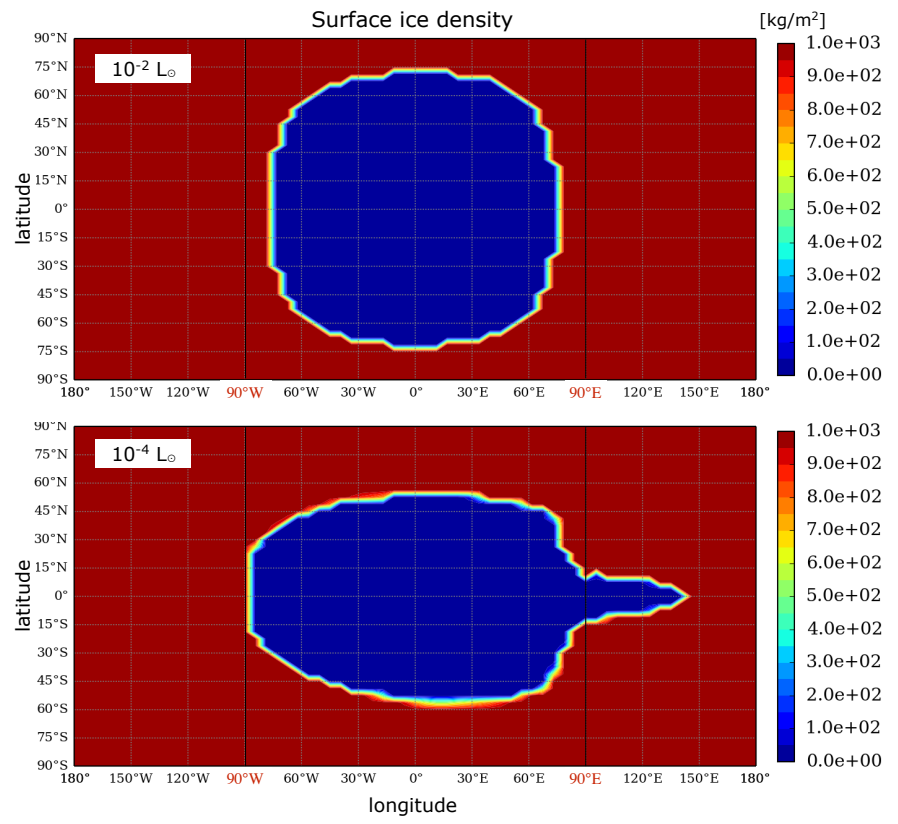

Fig. 4. Maps of the surface ice density for a planet on a circular orbit around a $10^{-2} L_{\odot}$ star (top) and a $10^{-4} L_{\odot}$ star (bottom). The map corresponding to the case $L_{\star}=1 L_{\odot}$ is in Fig. 1 .

and efficiently heat up the nightside of the planet. Figure 3 shows that the winds are much stronger for the case $10^{-4} L_{\odot}(\sim 50 \mathrm{~m} / \mathrm{s}$ vs. $\sim 25 \mathrm{~m} / \mathrm{s}$ for $10^{-2} L_{\odot}$ and $<10 \mathrm{~m} / \mathrm{s}$ for $\left.10^{-4} L_{\odot}\right)$ and redistribute the heat very efficiently along the equator. In this case, we note the presence of longitudinal wind jets at latitudes of $45^{\circ} \mathrm{S}$ and $45^{\circ} \mathrm{N}$ and at a pressure level of $\sim 30$ mbar. The wind velocity in the jets can reach almost $120 \mathrm{~km} / \mathrm{h}$. As the rotation period is of $\sim 2$ days, the criteria for the Rossby wave to be triggered is met and super-rotation takes place (Showman \& Polvani 2011; Leconte et al. 2013; Carone et al. 2015). As is true for $10^{-2} L_{\odot}$, the colder regions are the poles, but the temperature here is even lower (a surface temperature about $20 \mathrm{~K}$ lower than for $10^{-2} L_{\odot}$ ).

Figure 2 shows that this super-rotation also causes a longitudinal uniformity of the temperature, water ice clouds and water vapor distribution. Indeed, the nightside temperature of the lowest layer of the atmosphere for $10^{-4} L_{\odot}$ is not as low as for $1 L_{\odot}$ and $10^{-2} L_{\odot}$, and there are many more ice clouds and water vapor on the nightside for $10^{-4} L_{\odot}$ than for $1 L_{\odot}$ and $10^{-2} L_{\odot}$.

We note that the evolution of the surface ice density is not as quick as in the previous two cases where the surface ice density reaches its equilibrium in less than a few decades. Here a few thousands days are needed to reach equilibrium. One of the main differences it implies is an initially lower surface ice density for planets around $L_{\star}=10^{-4} L_{\odot}$ stars than for more luminous objects. First, the ocean's region survives and forms a belt around the equator with the belt buckle at the substellar point. As the eastward wind loses heat, the eastern regions are hotter than the western regions. After about 700 days of evolution, the ice forms a bridge at $120^{\circ}$ west, closing the equatorial ocean. Figure 4 shows that when equilibrium is reached, the shape of the ocean for $L_{\star}=10^{-4} L_{\odot}$ is very different from the shapes of the other two luminosities. When equilibrium is reached, we find that this planet has an ice-free ocean of a similar size to the previous cases, i.e., about $40 \%$ of the planet is ice-free.

Figure 5 shows the mean surface evolution and mean ice density evolution for planets around the three different host stars. For the circular orbits, we can see that the mean surface temperature 
is higher for $L_{\star}=10^{-4} L_{\odot}$ than for $L_{\star}=1 L_{\odot}$ and $L_{\star}=10^{-2} L_{\odot}$. This is due to the strong winds in the atmosphere of the planet, which chase the clouds that are forming above the substellar point. Without the cloud protection, the surface temperature increases. For a tidally locked planet orbiting very low luminosity objects, the longitudinal winds are strong and the stabilizing cloud feedback identified by Yang et al. (2013) is less efficient. Therefore, one might expect that the $\mathrm{HZ}$ of tidally locked planets around very low luminosity stars is not as extended as in Yang et al. (2013). However, simulations for higher incoming flux should be performed to verify this claim, in particular to see how the albedo varies with increasing incoming flux.

\section{Eccentric orbits}

As described in Sect. 3.2, we made sure that all planets receive on average the same flux as Earth. Increasing the eccentricity changes significantly the insolation the planet receives over one orbit and it leads to changes in the general characteristics of the climate. The insolation varies over the orbit and the substellar point moves along the equator as a result of the optical libration. Temperature, atmospheric water ice, atmospheric water vapor, and surface ice are influenced by this forcing, as shown in the following.

Figure 5 shows an example of the evolution, during two orbits, of the mean surface temperature and mean ice thickness for planets on a circular orbit and planets with an eccentricity of 0.2 and 0.4 , orbiting the three different object types. The mean surface temperature oscillates with the change of insolation over the orbit. As the orbital period changes with the luminosity and eccentricity (see Tables 2 and 3), it oscillates with different frequency and amplitude for the different cases. This oscillation of insolation and thus temperature has an effect on the amount of water vapor and surface ice (as seen in Fig. 5). We discuss in the following sections the impact on the climate of the planets considered here and the presence of liquid water at their surface.

\subsection{Luminosity of $L_{\star}=1 L_{\odot}$}

First, we consider the luminosity of a star similar to the luminosity of the Sun. First, for small eccentricities, our simulations show surface temperature oscillations, ice density, and water vapor oscillations. However, they remain small enough for the planet to keep surface liquid water. For example, a planet on an orbit of eccentricity 0.2 experiences temperature oscillations on the dayside of about $30 \mathrm{~K}$ in 371 days, while the mean temperature oscillations are of about $12 \mathrm{~K}$ (see Fig. 5). The surface ice density responds accordingly with the eccentricity-driven seasonal melting and freezing. On average, the surface ice density varies between $\sim 58 \%$ after the passage at periastron and $\sim 66 \%$ after the passage at apoastron. The region around the substellar point is always ice-free, but the center of the ice-free region shifts on the surface of the planet by about $10^{\circ}$ during the orbit.

Second, when we increase the eccentricity to 0.4 , the amplitude of the variations is higher. For example, Fig. 5 shows that the mean temperature variations increase from $\sim 12 \mathrm{~K}$ for an eccentricity of $e=0.2$ to $\sim 23 \mathrm{~K}$ for an eccentricity of $e=0.4$. Figure 5 also shows that the amplitude of the mean ice density variations of the planet also increases with the eccentricity. The planet's surface ice density after periastron is lower than for an eccentricity of $0.2(\sim 55 \%$ vs. $\sim 58 \%)$, but is much higher after apoastron ( $\sim 80 \%$ vs. $\sim 66 \%)$. The planet is never completely frozen during its evolution since an ice-free region always survives even after the passage at apoastron. Figure 6 shows the
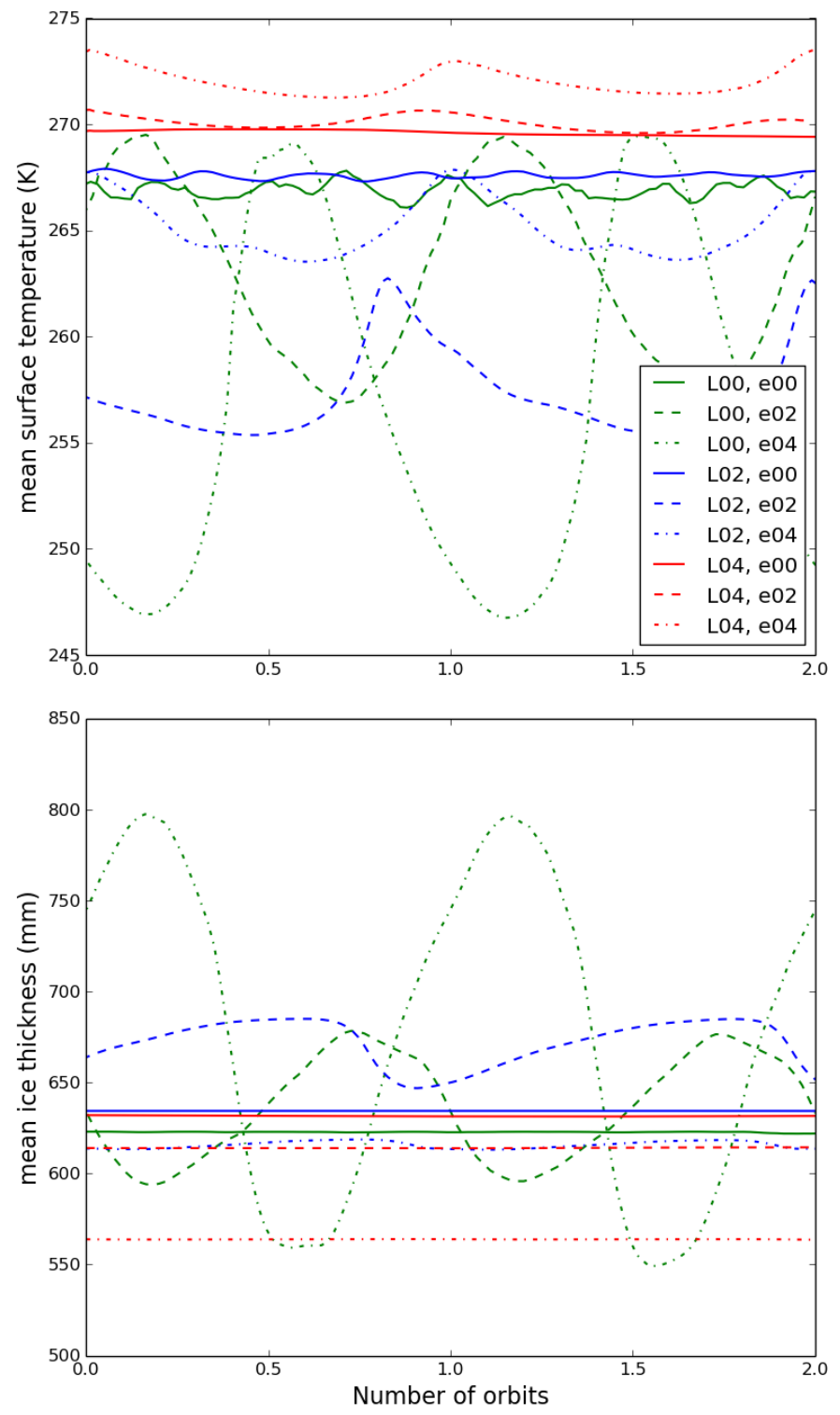

Fig. 5. Evolution over two orbits of the mean surface temperature and the mean ice thickness of a planet orbiting a $1 L_{\odot}$ star (L00, green), a $10^{-2} L_{\odot}$ star (L02, blue), and a $10^{-4} L_{\odot}$ (L04, red) star on an orbit of eccentricity 0.0 (e00), 0.2 (e02), and 0.4 (e04). We note that the orbital periods are different for each case (see Tables 2 and 3 ).

mean profiles of temperature, water ice, and water vapor in the atmosphere for different eccentricities. For non-zero eccentricities, the different quantities are plotted around apoastron and around periastron ${ }^{6}$. When the eccentricity increases, the apoastron and periastron profiles depart more from the circular mean profile. At apoastron, the temperature profile is colder than for $e=0$. There are fewer water ice clouds in the atmosphere and they are located closer to the surface. There is also less water vapor in the atmosphere. On the contrary, at periastron, the temperature profile is hotter, there are many more clouds located higher in the atmosphere, and there is also more water vapor in the atmosphere. For an eccentricity of 0.4 , the water vapor concentration in the upper atmosphere can reach a few $10^{-7} \mathrm{~kg} / \mathrm{m}^{2}$, which is about 7000 times higher that the water vapor concentration

6 Actually, it is a few days after the passage at periastron or apoastron, as there is a lag in the atmosphere's response time. We select the extreme values of the atmospheric water ice and vapor. 
a)

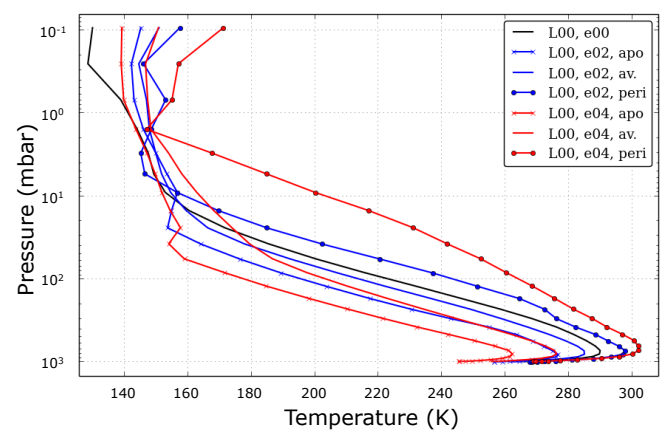

b)

Water ice profile

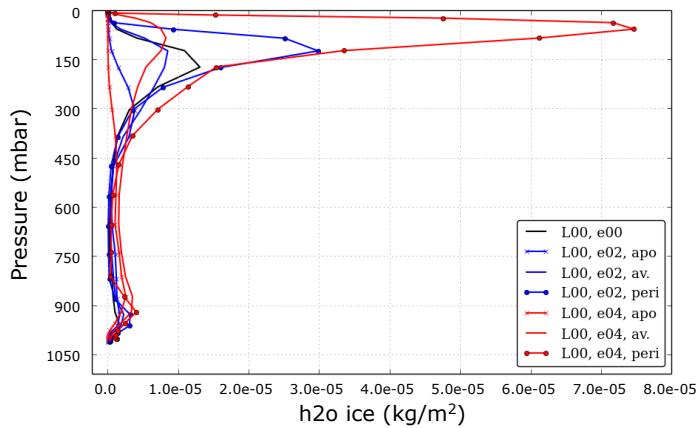

c) Water vapor profile

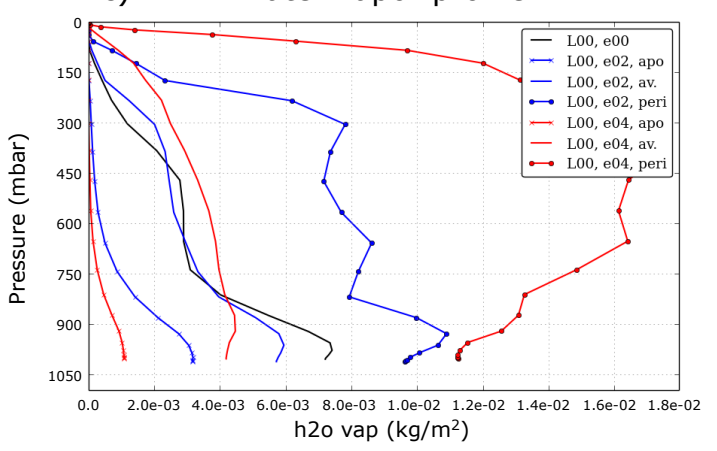

Fig. 6. Mean profiles of a) temperature (logarithmic scale); b) water ice; and c) water vapor (both in linear scale) in the atmosphere of a planet orbiting a $1 L_{\odot}$ star, on a circular orbit (black), an orbit of eccentricity 0.2 (blue), and 0.4 (red). For the eccentric cases, the temperature profiles at periastron (peri) and apoastron (apo) are represented, as well as the time average over one orbit (av.).

on Earth at the same altitude (Butcher et al. 1992). Owing to the passage at periastron where the planet can receive up to 2.5 times the insolation flux of the Earth, the water vapor peaks in the high atmosphere (pressure $<1$ mbar) for about 200 days above $10^{-8} \mathrm{~kg} / \mathrm{m}^{2}$, and for about 30 days above $10^{-7} \mathrm{~kg} / \mathrm{m}^{2}$. There is more water in the upper atmosphere for a planet on an orbit of eccentricity of 0.4 than there is in the upper atmosphere of a planet on a circular orbit.

We thus expect atmospheric loss to be more important for a planet on an eccentric orbit than a planet on a circular orbit. This process happens faster at periastron where the star-planet distance is shorter, which also coincides with the moment when the concentration of water vapor in the atmosphere is higher. We thus expect a larger atmospheric escape rate at periastron. However, as atmospheric escape happens on very long timescales, it might be sensitive only to an average value of the water vapor concentration and the difference of water vapor concentration at periastron or apoastron does not matter.

Third, for eccentricities higher than 0.6 , the planet is completely frozen around apoastron (corresponding to a mean ice thickness of $1 \mathrm{~m}$, our value of $h_{\text {ice }}$.). Figure 7 shows the evolution of the mean ice thickness for the three different host bodies. For an eccentricity of 0.6 , the orbital period is 432 days and the planet spends $\sim 100$ days in a completely frozen state, which corresponds to about $20 \%$ of its orbital period. When the planet gets closer to periastron, the ice starts melting at a longitude of $60^{\circ}$ west and liquid water is again available on the dayside around periastron. However, the water vapor concentration becomes very high and the surface temperature also increases to more than $300 \mathrm{~K}$.

In conclusion, for planets with an eccentricity of less than 0.6 , the planet is always able to sustain surface liquid water. The

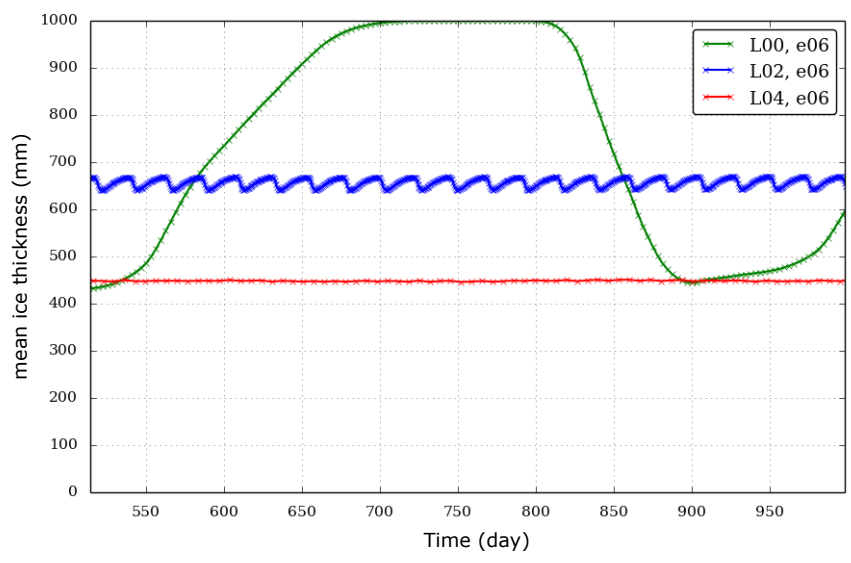

Fig. 7. Evolution of the mean ice thickness (here a thickness of $1000 \mathrm{~mm}$ means the whole planet is covered by the maximum depth of ice allowed in our model: $h_{\text {ice }}=1 \mathrm{~m}$ ) of a planet orbiting a $1 L_{\odot}$ star (green), a $10^{-2} L_{\odot}$ star (blue), and a $10^{-4} L_{\odot}$ star (red) on an orbit of eccentricity 0.6 .

ice-free region changes and shifts as the planet revolves around the star, but it never disappears. For planets with an eccentricity above 0.6, the planet cannot sustain surface liquid water during the whole orbital period. We note that for very high eccentricities and around periastron, the temperatures become higher than the model allows $(>400 \mathrm{~K})$. The mean flux approximation is therefore less valid for planets orbiting Sun-like stars on very high eccentricity orbits. However, departing from our definition of habitability based on sea-ice cover, we can only speculate how a potential life form on such a planet would survive this 


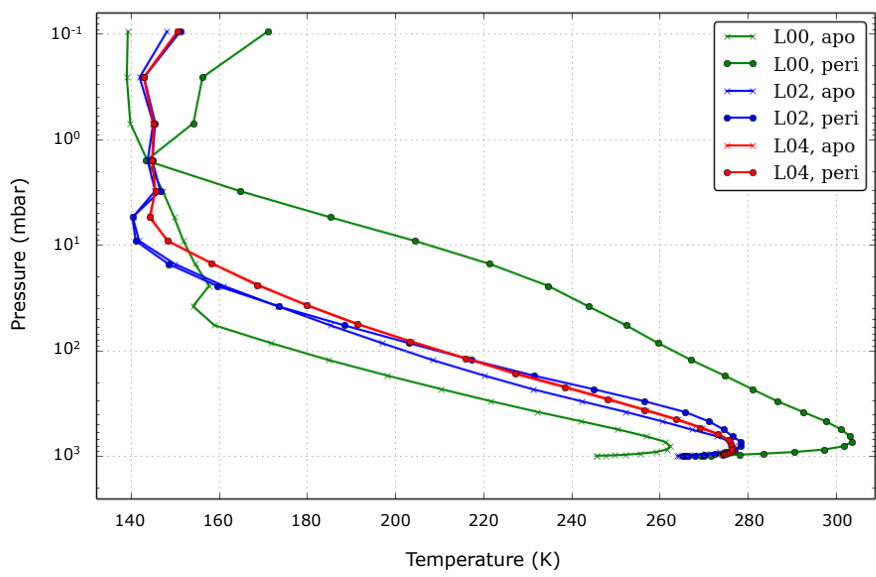

Fig. 8. Mean atmospheric temperature profile for planets with an eccentricity of 0.4 , orbiting a star of $1 L_{\odot}$ (green), $10^{-2} L_{\odot}$ (blue), and $10^{-4} L_{\odot}$ (red) for periastron and apoastron. We note that the two red curves are superimposed.

succession of frozen winters around apoastron and hot summers around periastron.

\subsection{Decreasing the luminosity}

Decreasing the luminosity has the effect of decreasing the eccentricity-driven insolation oscillation period. The insolation varies on a shorter timescale and this affects the climate response. The lower the luminosity, the less time the climate has to respond to the forcing.

\subsubsection{Luminosity of $L_{\star}=10^{-2} L_{\odot}$}

For a planet orbiting a $10^{-2} L_{\odot}$ star with an eccentricity of 0.2 , the dayside experiences temperature fluctuations of $\sim 40 \mathrm{~K}$ in about 23 days. It is of the same order of magnitude as for a $1 L_{\odot}$ star, but the fluctuation happens on a much shorter timescale. We note that decreasing the luminosity was done by decreasing the orbital period (the planets have to be closer to receive a flux of $1366 \mathrm{~W} / \mathrm{m}^{2}$ ), and as the planets are synchronized, the rotation period decreases as well (see Table 3). We did not take into account the spectral dependance of the low luminosity stars.

However, the oscillations of the average quantities have a much smaller amplitude than for $1 L_{\odot}$. Figure 5 shows that the amplitudes of oscillations of mean surface temperature and mean ice thickness are significantly damped when decreasing the luminosity from $1 L_{\odot}$ to $10^{-2} L_{\odot}$. Owing to the shorter orbital period, the frequency of the forcing is higher than for the case $1 L_{\odot}$ and the oscillations in the mean temperature, mean ice thickness, and mean water vapor concentration have a lower amplitude. The climate has indeed less time to react to the insolation forcing.

Figure 8 shows the mean atmospheric temperature profile for a planet at periastron and apoastron of an orbit of eccentricity 0.4 around the different kinds of object. The difference between apoastron and periastron is important for the case $1 L_{\odot}$ with a difference of about $40 \mathrm{~K}$ at a pressure level of $800 \mathrm{mbar}$. However, for $10^{-2} L_{\odot}$ the difference is negligible: a few kelvins from a pressure level of $800 \mathrm{mbar}$ to $50 \mathrm{mbar}$ and in the upper atmosphere (pressure $<1$ mbar). This shows how slowly the climate responds to the higher-frequency forcing.

Decreasing the luminosity has the effect of pushing the limit of liquid water presence toward higher eccentricities. For
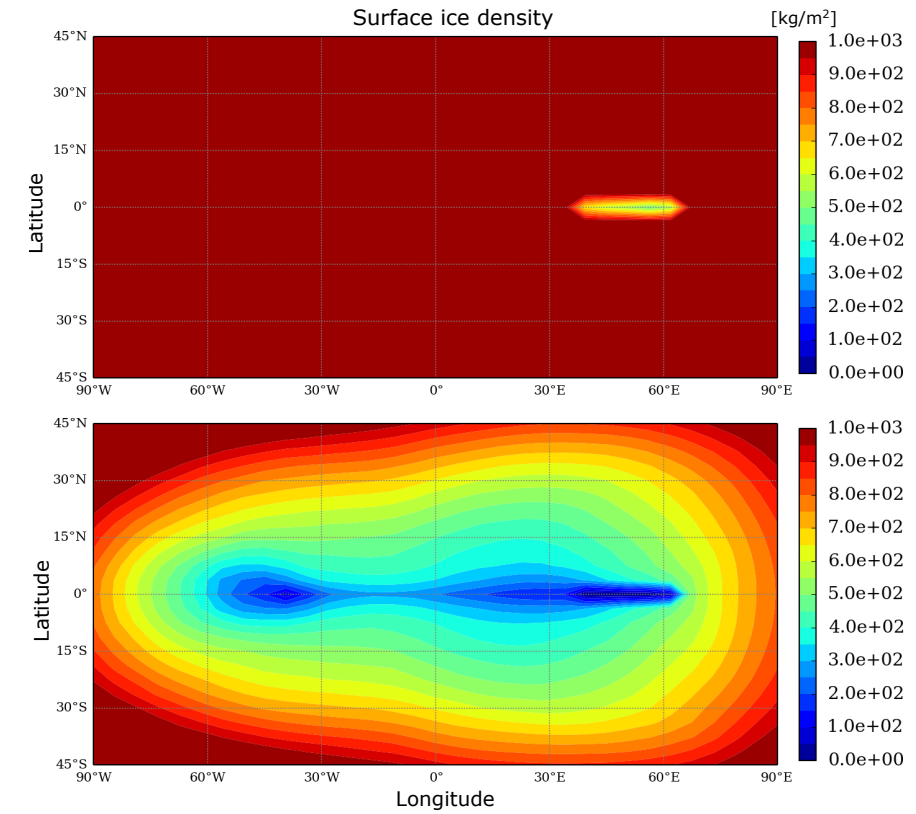

Fig. 9. Surface ice density for a planet of eccentricity 0.8 orbiting a $10^{-2} L_{\odot}$ star. Top graph: when the surface ice density is at its maximum (just before the periastron passage, i.e., after the long eccentricityinduced winter); bottom graph: when the surface ice density is at its minimum (just after the periastron passage).

example, Figure 7 shows that a planet with an eccentricity of 0.6 orbiting a $10^{-2} L_{\odot}$ star can always sustain surface liquid water whereas it cannot do so for the whole orbital period if it orbits a $1 L_{\odot}$ star.

For an eccentricity of 0.8 , the planet passes by a complete frozen state around apoastron and partially melts around periastron so that for about ten days $(\sim 1 / 3$ of the orbit) there is a small oblong ice-free region. This state is reached in $\sim 7500$ days. Figure 9 shows the surface ice density on the planet around periastron. Just after periastron, less than a percent of the planet's surface is ice-free at the equator around a longitude of $50^{\circ} \mathrm{E}$ (with an extent of about ten degrees in longitude and less than a degree in latitude, see bottom panel of Fig. 9). Just before periastron, i.e., after the long eccentricity-induced winter, the region that was ice-free around periastron freezes, but the ice layer depth does not reach its maximum value of $1 \mathrm{~m}$ (see top panel of Fig. 9). However, for an eccentricity of 0.9 , the planet rapidly becomes completely frozen. This state is reached at the first passage at apoastron where the planet freezes completely $-1 \mathrm{~m}$ of ice covering the whole planet - and melts partially around periastron. However, even around periastron there is always a thin layer of ice covering the whole planet.

As a result, we find that planets orbiting a $10^{-2} L_{\odot}$ star can always sustain surface liquid water on the dayside for higher eccentricities than for a $1 L_{\odot}$ star (up to 0.8 , instead of 0.6 ). For an eccentricity of 0.8 and higher, the planet remains completely frozen. All in all, the climate simulations are more in agreement with the mean flux approximation for planets orbiting $10^{-2} L_{\odot}$ stars. This is due to the averaging of the climate caused by the quicker rotation. However, departing from our definition of habitability based on sea-ice cover, we could speculate how a potential life form on such a planet would survive this rapid succession of frozen winters and hot summers (several tens of Kelvins in only $\sim 20$ days). 


\subsubsection{Luminosity of $L_{\star}=10^{-4} L_{\odot}$}

For an even less luminous host body, the amplitude of the oscillations in mean temperature and mean ice thickness are damped with respect to the other cases. As the duration of the year is very short ( 2 to 4 days, as specified in Table 3 ) there is a more effective averaging of the mean surface temperature and ice thickness than for longer orbital period planets. Figure 8 shows the mean temperature profile for planets at periastron and apoastron of an orbit of eccentricity 0.4 . For the case of $10^{-4} L_{\odot}$, there is no difference between periastron and apoastron. The shape of the surface ice density also changes when the luminosity decreases. For $10^{-2} L_{\odot}$, the ice-free region has a oblong shape, for $10^{-4} L_{\odot}$ it has a more peanut shape owing to the passage at periastron. The mean ice thickness for $L_{\star}=10^{-4} L_{\odot}$ is similar to the two previous cases, but does not significantly vary over time (see Fig. 7).

We find that planets orbiting a $10^{-4} L_{\odot}$ star always remain able to sustain surface liquid water on the dayside throughout the orbit up to very high eccentricities $(0.9$, instead of 0.8 for $10^{-2} L_{\odot}$ and 0.6 for $1 L_{\odot}$ ) owing to the efficient averaging of the climate brought about by the rapid rotation. The mean flux approximation is therefore valid for small luminosities. However, for an eccentricity of 0.8 , the dayside temperature varies over $\sim 50 \mathrm{~K}$, between $300 \mathrm{~K}$ and $350 \mathrm{~K}$ in less than 4 days. This situation is very extreme for potential life so that the question remains of how life could appear in such an unstable environment.

\section{Changing the thermal inertia of the oceans $I_{\text {oc }}$ and the ice thickness $h_{\text {ice }}$}

Changing the thermal inertia of the oceans or the ice thickness modifies a few properties of the planets' climate, but the consequences for surface liquid water remain basically the same. The time needed to reach equilibrium does not change significantly when increasing the thermal inertia of the ocean from $18000 \mathrm{~J} \mathrm{~s}^{-1 / 2} \mathrm{~m}^{-2} \mathrm{~K}^{-1}$ to $36000 \mathrm{~J} \mathrm{~s}^{-1 / 2} \mathrm{~m}^{-2} \mathrm{~K}^{-1}$, but - as Fig. 10 shows - it increases when increasing the ice thickness from $1 \mathrm{~m}$ to $10 \mathrm{~m}$. As the model is allowed to create thicker ice layers, more time is needed to reach the equilibrium ice thickness.

For planets orbiting a $1 L_{\odot}$ star on a circular orbit, changing the thermal inertia does not influence the overall evolution. The mean surface temperature evolves similarly for thermal inertia, and the equilibrium value is slightly higher for $I_{\mathrm{oc}}=18000 \mathrm{~J} \mathrm{~s}^{-1 / 2} \mathrm{~m}^{-2} \mathrm{~K}^{-1}$. There is more ice present for $I_{\mathrm{oc}}=$ $18000 \mathrm{~J} \mathrm{~s}^{-1 / 2} \mathrm{~m}^{-2} \mathrm{~K}^{-1}$ than for $I_{\mathrm{oc}}=36000 \mathrm{~J} \mathrm{~s}^{-1 / 2} \mathrm{~m}^{-2} \mathrm{~K}^{-1}$. When increasing the eccentricity, the main difference is that the oscillation amplitude in mean surface temperature and mean ice thickness is lower for $I_{\mathrm{oc}}=36000 \mathrm{~J} \mathrm{~s}^{-1 / 2} \mathrm{~m}^{-2} \mathrm{~K}^{-1}$. For an eccentricity of 0.4 , the mean surface temperature fluctuations amplitude is divided by two when multiplying the thermal inertia by two.

With a higher thermal inertia, the oceans can damp the climate fluctuations more efficiently. Although the temperature fluctuations on the dayside of the planet are still important, the extent of the ocean varies less with $I_{\mathrm{oc}}=36000 \mathrm{~J} \mathrm{~s}^{-1 / 2} \mathrm{~m}^{-2} \mathrm{~K}^{-1}$ than with $I_{\mathrm{oc}}=18000 \mathrm{~J} \mathrm{~s}^{-1 / 2} \mathrm{~m}^{-2} \mathrm{~K}^{-1}$, which makes the environment more stable for potential life. However, this higher thermal inertia of the oceans does not prevent the planet from being completely frozen for very eccentric orbits. For example, for $e=0.8$, the planet freezes completely at apoastron.

For planets orbiting a $10^{-2} L_{\odot}$ star on a circular orbit, the results are similar to the case $1 L_{\odot}$. However, the damping of the oscillation amplitude is not as pronounced. Thanks to the faster rotation of the planet and the more efficient heat redistribution,
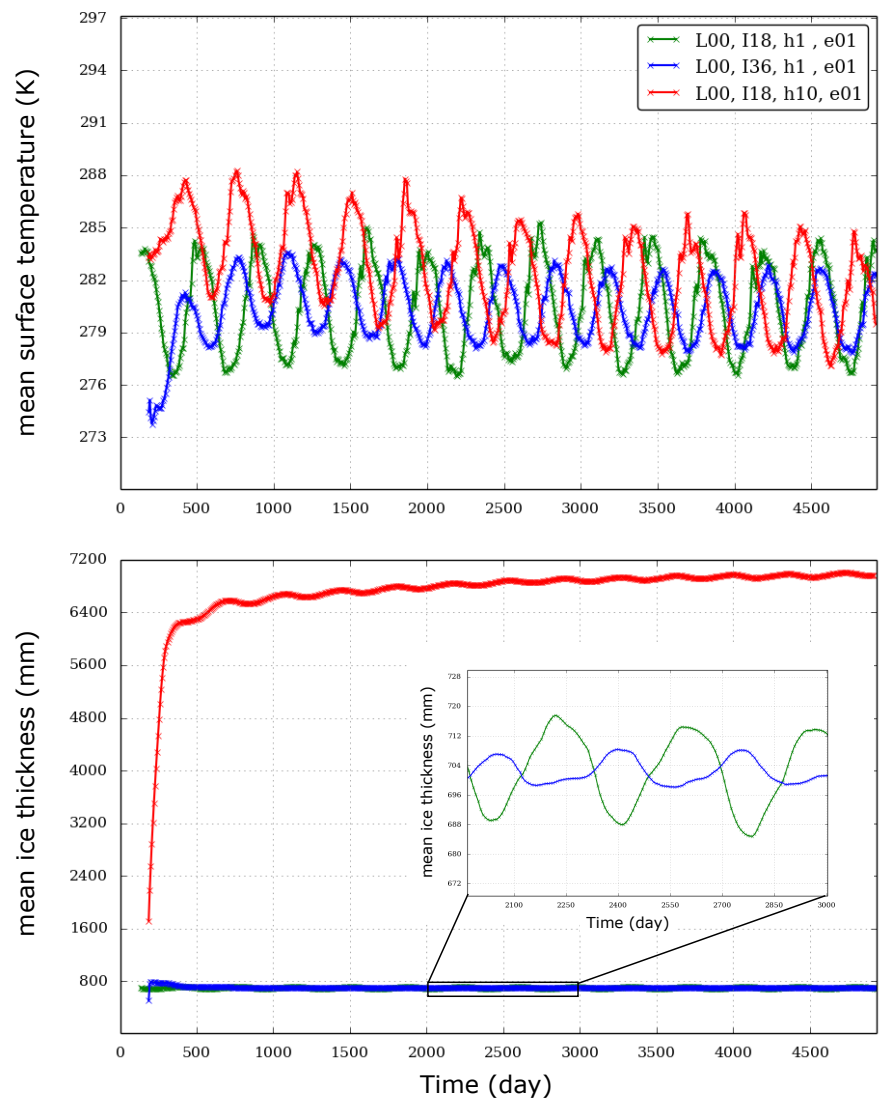

Fig. 10. Mean surface temperature and mean ice thickness of a planet orbiting a $1 L_{\odot}$ star with an eccentricity of 0.1 . Our standard scenario $I_{\mathrm{oc}}=18000 \mathrm{~J} \mathrm{~s}^{-1 / 2} \mathrm{~m}^{-2} \mathrm{~K}^{-1}, h_{\text {ice }}=1 \mathrm{~m}$ is in green; $I_{\mathrm{oc}}=36000 \mathrm{~J} \mathrm{~s}^{-1 / 2} \mathrm{~m}^{-2} \mathrm{~K}^{-1}, h_{\mathrm{ice}}=1 \mathrm{~m}$ in blue; and $I_{\mathrm{oc}}=$ $18000 \mathrm{~J} \mathrm{~s}^{-1 / 2} \mathrm{~m}^{-2} \mathrm{~K}^{-1}, h_{\text {ice }}=10 \mathrm{~m}$ in red.

the planet climate's is efficiently averaged and is less sensitive to the difference in thermal inertia.

Increasing the thermal inertia of the ocean allows the climate to have less extreme variations. Following our definition of habitability, changing the thermal inertia of the oceans does not influence the results. While the planet is able to sustain surface liquid water on the dayside for $I_{\mathrm{oc}}=18000 \mathrm{~J} \mathrm{~s}^{-1 / 2} \mathrm{~m}^{-2} \mathrm{~K}^{-1}$, it can still do so for $I_{\mathrm{oc}}=36000 \mathrm{~J} \mathrm{~s}^{-1 / 2} \mathrm{~m}^{-2} \mathrm{~K}^{-1}$. While the planet is only able to temporarily sustain surface liquid water on the dayside around periastron for $I_{\mathrm{oc}}=18000 \mathrm{~J} \mathrm{~s}^{-1 / 2} \mathrm{~m}^{-2} \mathrm{~K}^{-1}$, it is also the case for $I_{\mathrm{oc}}=36000 \mathrm{~J} \mathrm{~s}^{-1 / 2} \mathrm{~m}^{-2} \mathrm{~K}^{-1}$.

For planets orbiting a $1 L_{\odot}$ star on a circular orbit, changing the maximum ice thickness, $h_{\text {ice }}$, allowed in the model does not changes the equilibrium temperature of the planet (see Fig. 10), the amount of water vapor in the atmosphere, or the geographical repartition ocean-ice. However, more time is needed to reach equilibrium because a thicker ice layer has to be created.

For eccentric orbits, the eccentricity-driven oscillations of mean surface temperature and mean water vapor content are not damped with respect to the case $h_{\text {ice }}=1 \mathrm{~m}$. However, the shape of the ocean region remains very similar throughout the eccentric orbit. The ice layer on the border of the ice-free zone located in the western and eastern regions, which receives a strong insolation when the planet passes by periastron, only has time to partially melt. The ice-free zone is therefore smaller than for $h_{\text {ice }}=1 \mathrm{~m}$. 


\section{Observables}

The orbital phase curves of eccentric exoplanets have already been observed (e.g., the close-in giant planets HAT-P-2B by Lewis et al. 2013, HD 80606 by Laughlin et al. 2009, and WASP-14b by Wong et al. 2015) and modeled (e.g., Iro \& Deming 2010; Lewis et al. 2010, 2014; Kane \& Gelino 2011; Cowan \& Agol 2011; Selsis et al. 2013; Kataria et al. 2013). We show here that the variability caused by the eccentricity can be observed by orbital photometry in the visible and in the thermal infrared.

The model computes the top-of-the-atmopshere (TOA) outgoing flux in each spectral band and for each of the $64 \times$ 48 columns. The flux received from the planet by a distant observer at a distance $d$ in the spectral band $\Delta \lambda$ is given by

$\phi_{\Delta \lambda}(d)=\sum_{\text {lon,lat }} \frac{F_{\Delta \lambda, \text { lon,lat }}}{\pi} \times \frac{S_{\text {lat }} \mu_{\text {lon,lat }}}{d^{2}}$,

where the first term in the sum is the TOA scattered or emitted intensity, which is assumed to be isotropic, and the second term is the solid angle subtended by an individual cell; $S_{\text {lat }}$ is the area of the cell and $\mu_{\text {lon,lat }}$ is the cosine of the angle between the normal to the cell and the direction toward the observer. If this angle is negative then the cell is not on the observable hemisphere of the planet and we set $\mu_{\text {lon,lat }}=0$. In practice we use a suite of tools developed by Selsis et al. (2011) that can visualize maps of the emitted/scattered fluxes and produce time- or phase-dependent disk-integrated spectra at the spectral resolution of the GCM.

In addition to the inclination of the system, the orbital lightcurve of an eccentric planet also depends on the orientation of the orbit relative to the observer. Here we consider only observers in the plane of the orbit that see the planet in superior conjunction (full dayside) either at periastron or apoastron. In these configurations, a transit occurs at inferior conjunction and an eclipse at superior conjunction although these events are not included here. As shown by Selsis et al. (2011, 2013) and Maurin et al. (2012), lightcurves are only moderately sensitive to the inclination between $90^{\circ}$ (transit geometry) and $60^{\circ}$ (the median value). At an inclination of $0^{\circ}$ (polar observer), the phase angle is constant and the lightcurve is only modulated by the change of orbital distance. In general, the obliquity also introduces a seasonal modulation (Gaidos \& Williams 2004), but all our cases have a null obliquity. In this article we only describe observables obtained for the $1 L_{\odot}$ case. The idea is to illustrate the connection between climate and observables rather than to prepare the characterization of Earth-like planets around Sunlike stars as no forthcoming instrument will be able to provide such data. The James Webb Space Telescope (JWST), on the other hand, may be able to obtain (at a large observing-time cost) some data for terrestrial $\mathrm{HZ}$ planets around cool host-dwarfs (Cowan et al. 2015; Triaud et al. 2013; Belu et al. 2013). However, as before, without accounting for the actual spectral distribution of the host, which will be the object of a future study, the generated signatures of planets around $\mathrm{M}$ stars and brown dwarfs would not be realistic.

\subsection{Circular case}

Figure 11 was obtained for one orbit and a sub-observer point initially located arbitrarily on the equator. Each horizontal line of the plot represents the planet as seen by a distant observer at different orbital phases in a spectral band of the model (only the bands exhibiting a significant flux are shown, hence the gap between 2 and $6 \mu \mathrm{m}$ ). On the left side, the outgoing flux is shown

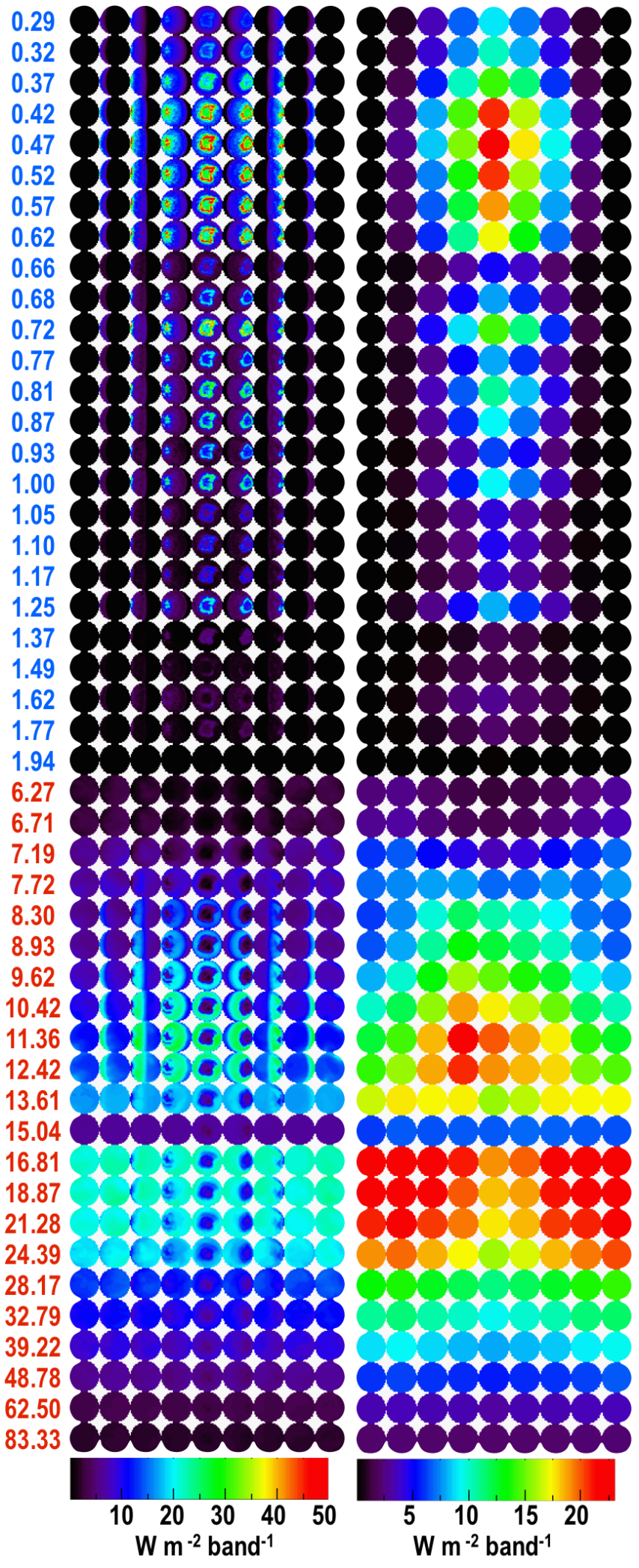

Fig. 11. Maps of TOA fluxes as seen by a distant observer for one given orbit and one observation geometry $\left(1 L_{\odot}\right.$, circular case). Each line represents one full orbit observed in one band (with the superior conjunction at the center). Each column represents a spectrum of the planet at the GCM resolution at a given orbital phase. The central wavelength of the bands is given in $\mu \mathrm{m}$ on the left. We note the gap between short (scattered light, in blue) and long (thermal emission, in red) wavelengths. In the right panel, the averaged spatially unresolved flux is given with a different color scale (owing to a smaller range of values). 

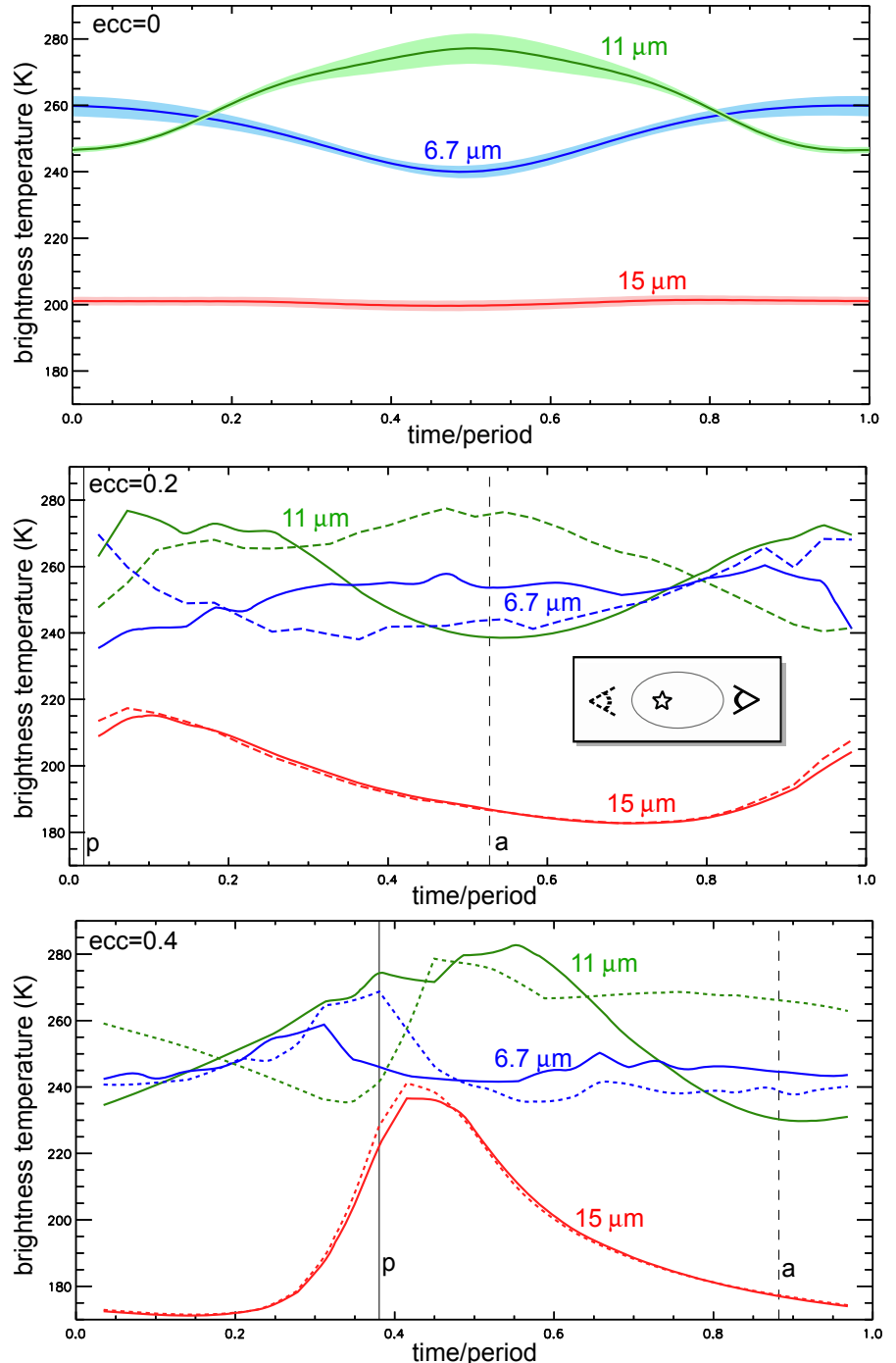

Fig. 12. Thermal phase curves in the $6.7,11$, and $15 \mu \mathrm{m}$ bands obtained for one orbit, for three eccentricities, and two observation geometries. In the circular case, lightcurves are centered on the superior conjunction (dayside in view) and are time-averaged while the gray area is the $1 \sigma$ variability due to meteorology. For a given eccentricity, the same orbit is used for both observation geometries. The periastron (p) and apoastron (a) are indicated by vertical lines.

at the spatial resolution of the GCM, while on the right side, the flux is a disk-average. In other words, the line in the right panel is a phase curve, while the column is an instantaneous disk-averaged spectrum. To obtain the flux measured per band at a distance $d$ the value given must be diluted by a factor $\left(R_{\oplus} / d\right)^{2}$. In addition to providing both spectral and photometric information, this type of figure is a useful tool for analyzing the energy budget of the planet: we can see where and at what wavelengths the planet receives its energy and cools to space. For the circular case, the top panel of Fig. 12 shows the thermal phase curves and their variability in bands centered on $6.7 \mu \mathrm{m}\left(\mathrm{H}_{2} \mathrm{O}\right.$ absorption), $11 \mu \mathrm{m}$ (window), and $15 \mu \mathrm{m}\left(\mathrm{CO}_{2}\right.$ absorption). The phase curve at $0.77 \mu \mathrm{m}$ (reflected light) and its variability are shown in Fig. 13 (black curve for the circular case). The long- and shortwave phase curves shown in Figs. 11-13 present several noticeable features:

- The central region of the dayside, which extends about $40^{\circ}$ from the substellar point, appears dark in the infrared owing

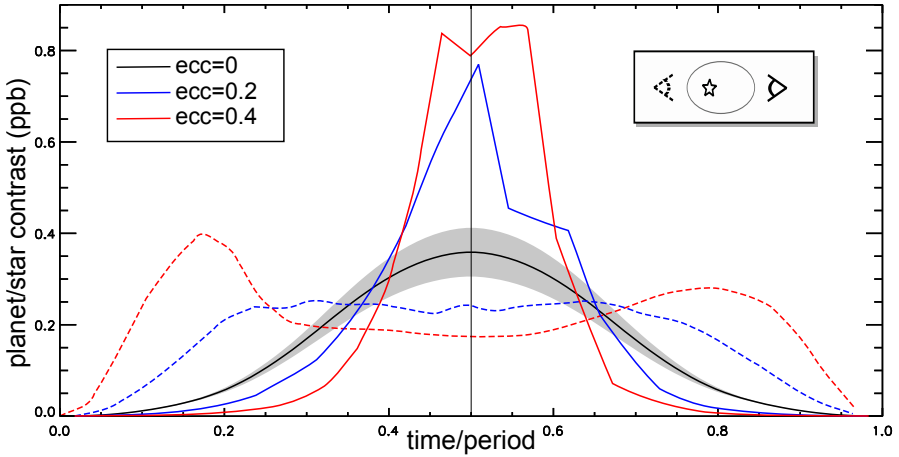

Fig. 13. Reflected-light phase curves in the $0.77 \mu \mathrm{m}$ band obtained for one orbit, for three eccentricities, and two observation geometries. In the circular case, lightcurves are centered on the superior conjunction (dayside in view) and are time-averaged while the gray area is the $1 \sigma$ variability due to meteorology. For a given eccentricity, the same orbit is used for both observation geometries, but unlike Fig. 12 they are shown here with half a period offset so that both are centered on the superior conjunction (vertical line).

to clouds and humidity associated with the massive updraft. At short wavelengths, this substellar cloudy area is instead the most reflective region owing to the scattering by clouds (see Fig. 11).

- As the modeled planet has a null obliquity and is locked in a 1:1 spin-orbit resonance, external forcing is constant at any given point on the planet. The three-dimensional structure of the planet that influences the emerging fluxes is not, however, constant. Clouds in particular produce stochastic meteorological phenomena that induce variations in the observables. The amplitude of this variability is shown in Fig. 13 for one visible band and in Fig. 12 for three infrared bands probing different levels: the surface $(11 \mu \mathrm{m})$, the lower and mid-atmospheres $\left(6.7 \mu \mathrm{m}, \mathrm{H}_{2} \mathrm{O}\right.$ band $)$, and the upper layers (15 $\mu \mathrm{m}, \mathrm{CO}_{2}$ band).

- The $\mathrm{CO}_{2}$ absorption band at $15 \mu \mathrm{m}$ band is the most noticeable spectral feature, but it exhibits no phase-variation because the atmospheric layers emitting to space in this band ( $~ 50-80$ mbar) are efficiently homogenized by circulation. This can be seen in Fig 2: the mean day and night thermal profiles are nearly identical in the range 400-20 mbar and clouds (on the dayside) do not extend at altitudes above the 100 mbar layer.

- Most of the cooling occurs within the 9-13 $\mu \mathrm{m}$ atmospheric window, which is transparent down to the surface in the absence of clouds, and between 16 and $24 \mu \mathrm{m}$, a domain affected by a significant $\mathrm{H}_{2} \mathrm{O}$ absorption increasing with the wavelength. In the 9-13 $\mu \mathrm{m}$ atmospheric window, most of the emission takes place on the cloud-free ring of the dayside. Except for the cloudy substellar region, the brightness temperature in this window is close to the surface temperature and thus goes from an average of around $270-280 \mathrm{~K}$ on the dayside down to $240-250 \mathrm{~K}$ on the nightside. In this window, the orbital lightcurve therefore peaks at superior conjunction, as we can see in the top panel of Fig. 12.

- At thermal wavelengths absorbed by water vapor $(5-7 \mu \mathrm{m}$ and above $16 \mu \mathrm{m}$ ) most of the emission takes place on the nightside, producing light curves that peak at inferior conjunction. The $6.7 \mu \mathrm{m}$ and $11 \mu \mathrm{m}$ lightcurves in the top panel of Fig. 12 are therefore in phase opposition. On the dayside, the large columns of water vapor result in a high altitude, and therefore a cold, $6.7 \mu \mathrm{m}$ photosphere (around $200 \mathrm{mbar}$, 
$240 \mathrm{~K})$. On the nightside, the $6.7 \mu \mathrm{m}$ photosphere is the surface owing to the low humidity (around 270-280 K).

- Yang et al. (2013) and Gómez-Leal (2013) noted that the wavelength-integrated emitted and scattered light have opposite phase variations. We also find this behavior because the emission at $\lambda>16 \mu \mathrm{m}$ represents the larger part of the bolometric cooling, as we can see in Fig. 11. However, the emission in the $9-13 \mu \mathrm{m}$ atmospheric band carries a significant fraction of the bolometric emission and peaks at superior conjunction, as reflected light does. A broadband that includes both spectral regions (9-13 and 16-25 $\mu \mathrm{m}$ ) therefore mixes two opposite phase variations, while distinguishing between these two domains of the thermal emission could provide strong constraints on the nature of the atmosphere. Although this should be explored further, these opposite phase variations between the 9-13 and 16-25 $\mu \mathrm{m}$ intervals may be a strong sign of both synchronization and the massive presence of water. Adequate filters at thermal wavelength could be used to exploit this signature while maximizing the signal-to-noise ratio.

\subsection{Eccentric cases}

The synthetic observables obtained for $1 L_{\odot}$ and $e=0.2$ and $e=0.4$ are given in Figs. 12 (infrared lightcurves), 13 (reflection lightcurves), and 15 (full orbital spectro-photometry). Because the maximum eccentricity of an observed possibly rocky planet is only 0.27 (see Sect. 1), we choose to show our cases up to an eccentricity of $e=0.4$. Several features can be noted:

- The emission in the $15 \mu \mathrm{m} \mathrm{CO}_{2}$ band remains phaseindependent owing to efficient heat redistribution at altitudes above the $100 \mathrm{mb}$ layer. As a consequence, the two observation geometries produce the same lightcurve, which vary only in response to the change of orbital distance (with a lag due to the inertia of the system). This is particularly noticeable in Fig. 12.

- The phase opposition that exists in the circular case between the scattered light and the emission in the $8-12 \mu \mathrm{m}$ atmospheric window on the one hand and the emission in the 16-25 $\mu \mathrm{m}$ water vapor window on the other hand disappears at $e=0.4$ and is hardly seen at $e=0.2$ except when the superior conjunction appears at apoastron (second panel in Fig. 15). This can be explained by the strong periastron warming that generates a cloudiness that covers a larger fraction of the sunlit hemisphere and persists on the nightside. These clouds hence decrease the infrared cooling in the atmospheric window on the dayside.

- Another effect of this large cloud coverage is to spread the observed scattered light over a broader range of phase angles, with no peak at superior conjunction when it occurs at apoastron. This can be seen in Figs. 13 and 15.

\subsection{Variation spectrum}

The variation spectrum, as defined in Selsis et al. (2011), is the peak amplitude of the phase curve as a function of wavelength. Figure 14 shows the minimum and maximum that can be observed at any time during a complete orbit for each band of the GCM. Two opposite geometries are presented, for $e=0,0.2$, and 0.4 and $L_{\star}=L_{\odot}$. The difference between the maximum and the minimum gives the amplitude spectrum at the resolution of the GCM. As we only consider cases with a $90^{\circ}$ inclination, the

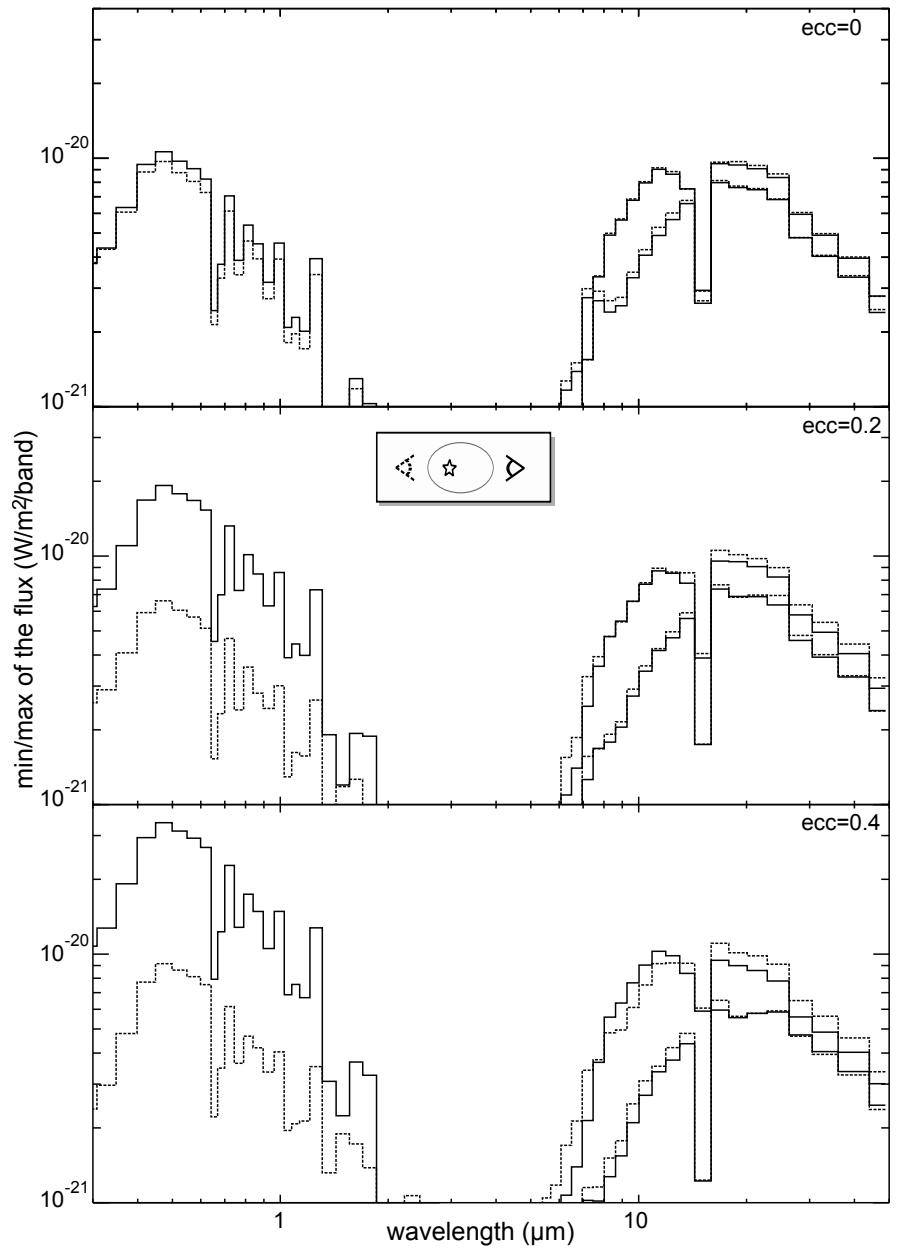

Fig. 14. Spectral variability. These graphs show, for $L_{\star}=L_{\odot}$ and $e=0$, 0.2 , and 0.4 , the maximum and minimum of the flux in each band received during one orbit. Flux is given at $10 \mathrm{pc}$. For the reflected light, the minimum is zero as the observer is in the plane of the orbit (inclination $=90^{\circ}$ ). Superior conjunction occurs at periastron for solid lines and at apoastron for dashed lines. For the circular case (top), solid and dashed lines indicate two arbitrary observer directions separated by $180^{\circ}$ and, in this case, the difference between the two curves is only due to stochastic meteorological variations.

reflected flux is always null at inferior conjunction (transit). The reflected variation spectrum naturally depends on the eccentricity: the closer the planet approaches its hot star, the higher its reflected brightness (for a given albedo). The thermal variation spectrum shows, on the other hand, an overall minor dependency on the eccentricity. The amplitude of the differences in thermal flux increases slightly with eccentricity, but the differences between one eccentricity and another are not significantly higher than those induced by stochastic meteorological changes from one orbit to another. One exception is the amplitude of the variations in the $15 \mu \mathrm{m}$ band, which increases notably with the eccentricity from basically no variations at $e=0$ to a factor of 2 variation at $e=0.2$ and a factor of 5 at $e=0.4$.

\subsection{Radiative budget}

An observer that would have the ability to measure the orbitaveraged broadband emission at short (SW: 0.25-2.0 $\mu \mathrm{m}$ ) and/or long (LW: 6-35 $\mu \mathrm{m}$ ) wavelengths would be able to estimate the Bond albedo. This could be done with either of these two values 
Table 4. Bond albedo computed and measured for the $1 L_{\odot}$ case.

\begin{tabular}{lccc}
\hline \hline$e$ & 0.0 & 0.2 & 0.4 \\
\hline$A_{\text {Bond }}$ & 0.25 & 0.295 & 0.35 \\
\hline$A_{\text {obs }}$ \\
Equatorial observer $\left(i=90^{\circ}\right)$ \\
SW & $0.26-0.29$ & $0.29-0.34$ & $0.34-0.43$ \\
LW & $0.27-0.29$ & $0.28-0.35$ & $0.34-0.42$ \\
LW/SW & $0.26-0.29$ & $0.29-0.35$ & $0.34-0.42$ \\
Polar observer $\left(i=0^{\circ}\right)$ & & \\
LW/SW & $0.22-0.23$ & $0.26-0.27$ & $0.32-0.34$ \\
\hline
\end{tabular}

Notes. $A_{\mathrm{obs}}$ is the Bond albedo estimated by a distant observer with broadband photometry (see text). The range of values results from changing the observing geometry and the meteorology. For the polar observer, we assume that the radius of the planet is unknown.

if the planetary radius and the incoming stellar flux are known, or from the LW/SW ratio without any other information. However, these estimates would be biased by the observing geometry and emission/reflection anisotropies. We therefore tested how much these measured values of the Bond albedo depart from its actual value. The actual Bond albedo calculated from the simulations and those that can be estimated by an observer are given in Table 4. We can see that the error is typically on the order of $10-20 \%$, with a slight tendency to overestimate the Bond albedo. This is occurs because the reflected light is more focused into the orbital plane than the thermal emission, which is re-distributed by circulation. A polar observer may instead underestimate the Bond albedo.

\section{Discussion}

We studied the influence of the duration of the orbital period and eccentricity on the climate of tidally locked ocean covered planets orbiting objects of different luminosities. We chose here to do a parametric study keeping most things equal between our simulations: we considered synchronized planets and we did not take into account the spectral differences between a Sun-like star and a lower luminosity object.

Low mass stars have a redder spectrum and this changes the albedo of the ice and snow (Joshi \& Haberle 2012; von Paris et al. 2013). Taking this phenomenon into account would radically change the ice-albedo feedback, so that for redder objects it would not be able to drive the planets into a glaciation state (as we showed here for planets with high eccentricities around a $L_{\star}=10^{-2} L_{\odot}$ star). Godolt et al. (2015) showed that for the same incoming flux, the planets orbiting redder objects would be hotter, and generally would have different climate states than those orbiting a $1 L_{\odot}$ star (see also Shields et al. 2014).

Our results would also have been affected had we taken into account a realistic oceanic circulation. Oceanic circulation has the effect of homogenizing the climate and facilitating the existence of habitable states. Godolt et al. (2015) shows that taking oceanic circulation into account produces surface habitable conditions for planets orbiting F-type stars instead of a snowball state. Our results would likely be different if we had considered other types of planets such as an Earth-like planet, a land planet, a planet with a Pangea-like continent, or a planet with archipelagos (e.g., Yang et al. 2014).
Furthermore, we consider here a planet whose atmosphere has a composition very similar to the Earth's. However, the atmospheric composition could be different. For instance, there could be more $\mathrm{CO}_{2}$, which would contribute to heating up the planet. Also, the pressure in the atmosphere could be different, which would affect the climatic response to eccentricity.

In this work we also made the choice of neglecting the effects of tides even though we considered high eccentricities. Tides would have several effects on the system considered here. First, tides act to damp the eccentricities of planets, so that a planet with an eccentricity of 0.8 would not keep its eccentricity forever (the timescale of eccentricity damping depending on many parameters such as the orbital parameters, the mass, radius, and composition of the planet; e.g., Mignard 1979; Hut 1981). However, a high eccentricity could be maintained if the planet is part of a multiplanet system. Indeed the gravitational interactions between the planets can contribute in exciting the eccentricity of the planet on long timescales. Second, such high eccentricities can be responsible for tidal heating in the interior of the planet (Jackson et al. 2008; Barnes et al. 2009; Bolmont et al. 2014). This tidal heating is all the more important as we consider planets orbiting low luminosity objects. Indeed, a planet around a less luminous star and receiving the same average flux as Earth is much closer to its host star and thus susceptible to a higher tidal heating. This effect should be studied, as tidal heating could contribute significantly to the energy budget of the planet (and create "tidal Venuses" as discussed in Barnes et al. 2009). Third, tides also influence the rotation state of the planet. We considered tidally locked planets here, but planets on eccentric orbits are more likely to have a pseudo-synchronous rotation (synchronization around periastron; see Hut 1981) or to be in spin-orbit resonance (the higher the eccentricity, the higher the order of the resonance; see Makarov \& Efroimsky 2013). The pseudosynchronous rotation and the spin-orbit resonance are faster than the synchronous rotation, so the wind will redistribute the heat on the planet even more efficiently. Finally, even for a circular orbit, atmospheric tides can drive a planet out of synchronization (Correia et al. 2003; Leconte et al. 2015).

In this work, we focused only on the presence of surface liquid water without having to determine the actual potential of the planets as appropriate environments for the appearance of life. Indeed, the question of life on water worlds is still open. In order for life to appear and evolve, water must be in contact with the building blocks of life, such as phosphorus, sulfur, iron, magnesium, and nitrogen. This is possible if the ocean is in direct contact with the planet's silicate mantle. However, Sotin et al. (2007) found that ocean planets are likely to have a high pressure ice layer between the liquid water ocean and the silicate mantle. Therefore, Lammer et al. (2009) classified the ocean planets as class IV habitats, the lowest class of potentially habitable worlds (see also Forget et al. 2013). However, Léger et al. (2004) and Forget et al. (2013) both highlighted the possibility of enriching the ocean with minerals from meteoritic impacts, showing that these planetary objects should not be discarded as potential life habitats. In addition, some other mechanisms were put forward to bring minerals to the liquid water layer, such as solid convection (e.g., the presence of ${ }^{36} \mathrm{Ar}$ at the surface of Titan could be explained by solid convection within the subsurface high pressure ice layer; Niemann et al. 2010; Tobie et al. 2006). Finally, Alibert (2015) showed that planets with a small mass fraction of water ( $\sim 1-2 \%$, depending on the mass of the planet) have more probability of being habitable because of the absence of a high pressure ice layer. 

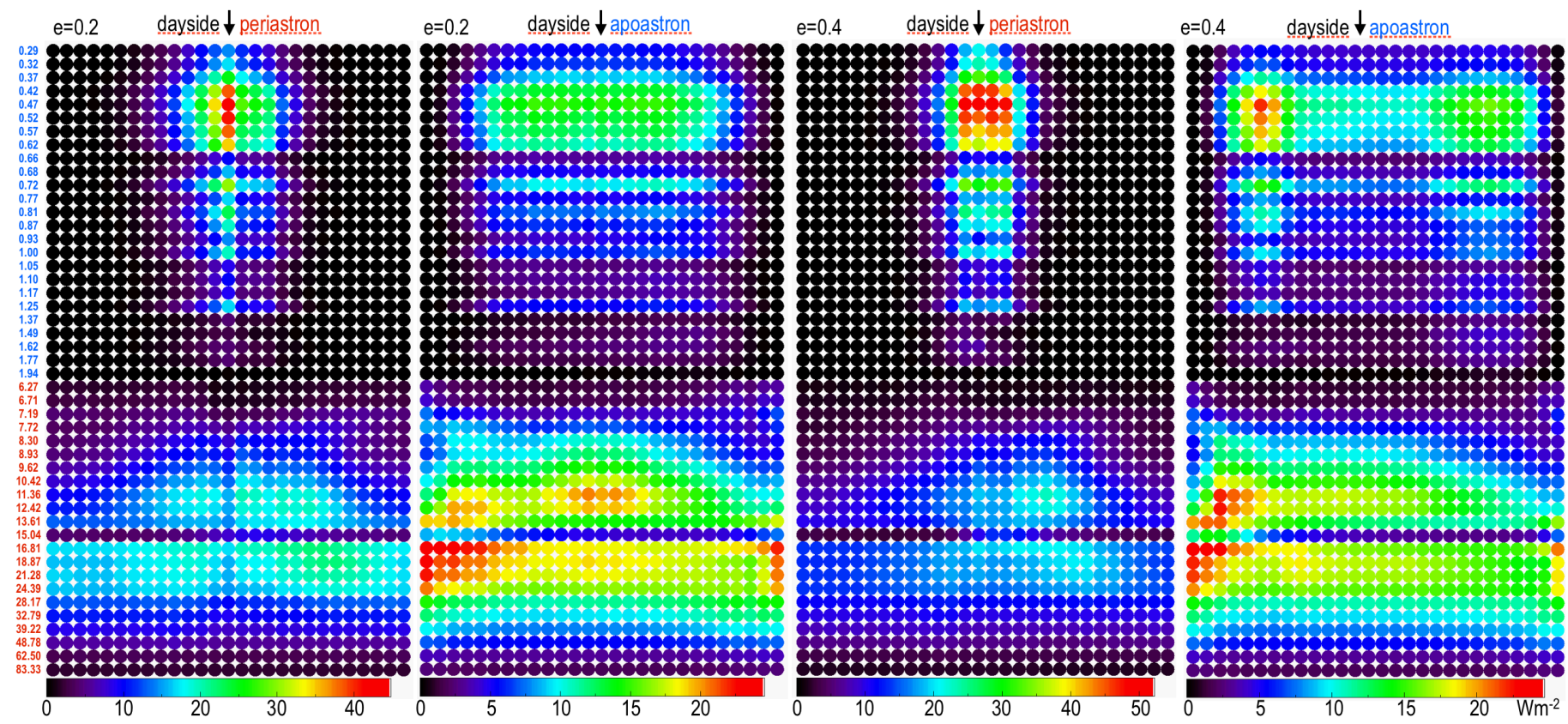

Fig. 15. Similar to the right panel of Fig. 11 but for eccentric cases. Two observation geometries are shown for each eccentricity: the arrows at the top indicate whether the dayside is observed at apoastron or periastron. The different color scales indicate the spatially unresolved flux.

\section{Conclusions}

Thanks to climate simulations of synchronous ocean covered planets, we are able to assess their potential habitability in the sense of their ability to host a liquid water ocean at their surface.

We investigated whether the mean flux approximation (Williams \& Pollard 2002) correctly assesses the habitability of eccentric planets orbiting stars of different luminosity. To this end, we considered planets receiving on average the same flux as Earth and modeled their climates for different eccentricities and different orbital periods (used here as a proxy for the three different star luminosities we considered: $1 L_{\odot}, 10^{-2} L_{\odot}, 10^{-4} L_{\odot}$ ).

We found that tidally locked water worlds can sustain surface liquid water only on the dayside. For all luminosities and only small eccentricities, all the planets considered can always sustain surface liquid water on the dayside. Planets orbiting the less luminous objects considered here $\left(10^{-4} L_{\odot}\right)$ can always sustain surface liquid water whatever the eccentricity.

However, planets on high eccentricity orbits around luminous objects can only sustain surface liquid water around periastron. This is the case for planets orbiting a $1 L_{\odot}$ star with an eccentricity higher than 0.6 , planets orbiting a $10^{-2} L_{\odot}$ star with an eccentricity higher than 0.8 , and planets orbiting a $10^{-4} L_{\odot}$ star with an eccentricity higher than 0.9 . For example, we find that a planet orbiting a $10^{-2} L_{\odot}$ star with an eccentricity of 0.9 cannot have surface liquid water; it always has an ice layer covering the whole planet. In addition, for planets with high eccentricities, the dayside temperature variations over a period of 365 days $\left(1 L_{\odot}\right)$ to 4 days $\left(10^{-4} L_{\odot}\right)$ can be huge (up to $100 \mathrm{~K}$ ). This could have detrimental consequences for eventual life forms. Figure 16 summarizes our results.

For the planets considered here, i.e., water world planets for which the surface is treated as an infinite water source, tidally locked, and with no obliquity, we found that the higher the eccentricity of the planet or the higher the luminosity of the star, the less reliable the mean flux approximation. These results are not in agreement with the work of Williams \& Pollard (2002), who determined that the mean flux approximation is valid for all

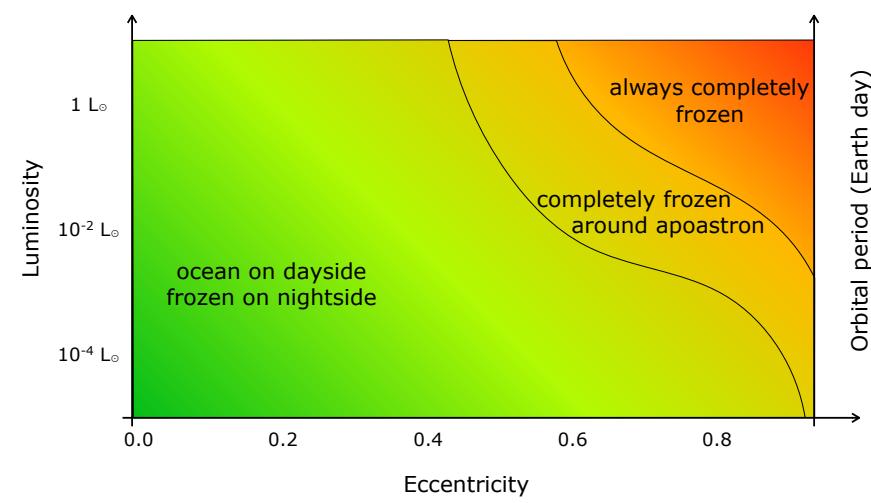

Fig. 16. Liquid water coverage map of tidally locked ocean-covered planets orbiting stars of different luminosities and with different orbital eccentricities.

eccentricities. When considering $L_{\star}=1 L_{\odot}$, we draw similar conclusions to those of Linsenmeier et al. (2015), who pointed out that planets on eccentric orbits around a Sun-like star can be frozen during part of the year.

We explored the parameter space of our model by changing the thermal inertia of the ocean and the maximum ice layer thickness. Changing those parameters in the model does not change our conclusions significantly.

We will address the limits discussed in the previous section in a future work, in particular the spectral difference between low mass stars and the Sun, the distribution of continents, and tidal effects.

Acknowledgements. This work was supported by the Fonds de la Recherche Scientifique-FNRS under Grant No. T.0029.13 ("ExtraOrDynHa" research project). Computational resources have been provided by the Consortium des Équipements de Calcul Intensif (CÉCI), funded by the Fonds de la Recherche Scientifique de Belgique (F.R.S.-FNRS) under Grant No.2.5020.11. The authors would like to thank Ehouarn Millour and Aymeric Spiga for their help in this work and Stephen Kane for the Habitable Zone Gallery. 


\section{References}

Alibert, Y. 2015, Origins of Life and Evolution of the Biosphere, 45, 319 Anglada-Escudé, G., Arriagada, P., Vogt, S. S., et al. 2012, ApJ, 751, L16 Bailey, J., Butler, R. P., Tinney, C. G., et al. 2009, ApJ, 690, 743

Barnes, R., Raymond, S. N., Jackson, B., \& Greenberg, R. 2008, Astrobiology, 8,557

Barnes, R., Jackson, B., Greenberg, R., \& Raymond, S. N. 2009, ApJ, 700, L30

Belu, A. R., Selsis, F., Raymond, S. N., et al. 2013, ApJ, 768, 125

Bolmont, E., Raymond, S. N., \& Selsis, F. 2014, in SF2A-2014: Proc. Annual meeting of the French Society of Astronomy and Astrophysics, eds. J. Ballet, F. Martins, F. Bournaud, R. Monier, \& C. Reylé, 63

Borucki, W. J., Koch, D. G., Basri, G., et al. 2011, ApJ, 736, 19

Butcher, S. S., Charlson, R. J., Orians, G. H., \& Wolfe, G. V. 1992, Global Biogeochemical Cycles (London: Academic Press)

Carone, L., Keppens, R., \& Decin, L. 2015, MNRAS, 453, 2412

Clough, S. A., Kneizys, F. X., \& Davies, R. W. 1989, Atmospheric Research, 23, 229

Correia, A. C. M., Laskar, J., \& de Surgy, O. N. 2003, Icarus, 163, 1

Cowan, N. B., \& Agol, E. 2011, ApJ, 726, 82

Cowan, N. B., Greene, T., Angerhausen, D., et al. 2015, PASP, 127, 311

Dressing, C. D., Spiegel, D. S., Scharf, C. A., Menou, K., \& Raymond, S. N. 2010, ApJ, 721, 1295

Ehrenreich, D., Bourrier, V., Wheatley, P. J., et al. 2015, Nature, 522, 459

Forget, F., Wordsworth, R., Millour, E., et al. 2013, Icarus, 222, 81

Gaidos, E., \& Williams, D. M. 2004, New Astron., 10, 67

Godolt, M., Grenfell, J. L., Hamann-Reinus, A., et al. 2015, Planet. Space Sci., 111,62

Gómez-Leal, I. 2013, Ph.D. Thesis, University of Bordeaux, France

Hourdin, F., Musat, I., Bony, S., et al. 2006, Climate Dynamics, 27, 787

Hut, P. 1981, A\&A, 99, 126

Iro, N., \& Deming, L. D. 2010, ApJ, 712, 218

Jackson, B., Greenberg, R., \& Barnes, R. 2008, ApJ, 681, 1631

Joshi, M. M., \& Haberle, R. M. 2012, Astrobiology, 12, 3

Kaltenegger, L., Sasselov, D., \& Rugheimer, S. 2013, ApJ, 775, L47

Kane, S. R., \& Gelino, D. M. 2011, ApJ, 741, 52

Kane, S. R., \& Gelino, D. M. 2012, PASP, 124, 323

Kasting, J. F., Whitmire, D. P., \& Reynolds, R. T. 1993, Icarus, 101, 108

Kataria, T., Showman, A. P., Lewis, N. K., et al. 2013, ApJ, 767, 76

Kopparapu, R. K. 2013, ApJ, 767, L8

Kopparapu, R. K., Ramirez, R. M., SchottelKotte, J., et al. 2014, ApJ, 787, L29

Kuchner, M. J. 2003, ApJ, 596, L105

Kulikov, Y. N., Lammer, H., Lichtenegger, H. I. M., et al. 2007, Space Sci. Rev., 129,207

Lammer, H., Selsis, F., Ribas, I., et al. 2003, ApJ, 598, L121

Lammer, H., Bredehöft, J. H., Coustenis, A., et al. 2009, A\&ARv, 17, 181

Laughlin, G., Deming, D., Langton, J., et al. 2009, Nature, 457, 562

Leconte, J., Forget, F., Charnay, B., et al. 2013, A\&A, 554, A69
Leconte, J., Wu, H., Menou, K., \& Murray, N. 2015, Science, 347, 632

Léger, A., Selsis, F., Sotin, C., et al. 2004, Icarus, 169, 499

Lewis, N. K., Showman, A. P., Fortney, J. J., et al. 2010, ApJ, 720, 344

Lewis, N. K., Knutson, H. A., Showman, A. P., et al. 2013, ApJ, 766, 95

Lewis, N. K., Showman, A. P., Fortney, J. J., Knutson, H. A., \& Marley, M. S. 2014, ApJ, 795, 150

Linsenmeier, M., Pascale, S., \& Lucarini, V. 2015, Planet. Space Sci., 105, 43

Makarov, V. V., \& Efroimsky, M. 2013, ApJ, 764, 27

Manabe, S., \& Wetherald, R. T. 1967, J. Atmospheric Sciences, 24, 241

Maurin, A. S., Selsis, F., Hersant, F., \& Belu, A. 2012, A\&A, 538, A95

Mignard, F. 1979, Moon and Planets, 20, 301

Milankovitch, M. 1941, Kanon der Erdebestrahlung und seine anwendung auf das eiszeitenproblem (Koniglich Serbische Akademie)

Niemann, H. B., Atreya, S. K., Demick, J. E., et al. 2010, J. Geophys. Res., 115, 12006

Pierrehumbert, R. T. 2011, ApJ, 726, L8

Quintana, E. V., Barclay, T., Raymond, S. N., et al. 2014, Science, 767, 128

Robertson, P., \& Mahadevan, S. 2014, ApJ, 793, L24

Rothman, L. S., Gordon, I. E., Barbe, A., et al. 2009, J. Quant. Spectr. Rad. Transf., 110, 533

Selsis, F., Kasting, J. F., Levrard, B., et al. 2007, A\&A, 476, 1373

Selsis, F., Wordsworth, R. D., \& Forget, F. 2011, A\&A, 532, A1

Selsis, F., Maurin, A.-S., Hersant, F., et al. 2013, A\&A, 555, A51

Shields, A. L., Meadows, V. S., Bitz, C. M., et al. 2013, Astrobiology, 13, 715

Shields, A. L., Bitz, C. M., Meadows, V. S., Joshi, M. M., \& Robinson, T. D. 2014, ApJ, 785, L9

Showman, A. P., \& Polvani, L. M. 2011, ApJ, 738, 71

Showman, A. P., Fortney, J. J., Lewis, N. K., \& Shabram, M. 2013, ApJ, 762, 24

Showman, A. P., Lewis, N. K., \& Fortney, J. J. 2015, ApJ, 801, 95

Sotin, C., Grasset, O., \& Mocquet, A. 2007, Icarus, 191, 337

Spiegel, D. S., Menou, K., \& Scharf, C. A. 2008, ApJ, 681, 1609

Spiegel, D. S., Burrows, A., \& Milsom, J. A. 2010, in AAS/Division for Planetary Sciences Meeting Abstracts, 42, 27.27

Tobie, G., Lunine, J. I., \& Sotin, C. 2006, Nature, 440, 61

Triaud, A. H. M. J., Gillon, M., Selsis, F., et al. 2013, ArXiv e-prints [arXiv: 1304.7248]

von Paris, P., Selsis, F., Kitzmann, D., \& Rauer, H. 2013, Astrobiology, 13, 899

Williams, D. M., \& Kasting, J. F. 1996, in Lunar and Planetary Science Conference, 27, 1437

Williams, D. M., \& Kasting, J. F. 1997, Icarus, 129, 254

Williams, D. M., \& Pollard, D. 2002, Int. J. Astrobiol., 1, 61

Wong, I., Knutson, H. A., Lewis, N. K., et al. 2015, ApJ, 811, 122

Wordsworth, R. D., Forget, F., Selsis, F., et al. 2010, A\&A, 522, A22

Wordsworth, R. D., Forget, F., Selsis, F., et al. 2011, ApJ, 733, L48

Wordsworth, R., Forget, F., Millour, E., et al. 2013, Icarus, 222, 1

Yang, J., Cowan, N. B., \& Abbot, D. S. 2013, ApJ, 771, L45

Yang, J., Liu, Y., Hu, Y., \& Abbot, D. S. 2014, ApJ, 796, L22 